

\title{
Effective response of classical, auxetic and chiral magnetoelastic materials by use of a new variational principle
}

Kostas Danas

\section{- To cite this version:}

Kostas Danas. Effective response of classical, auxetic and chiral magnetoelastic materials by use of a new variational principle. Journal of the Mechanics and Physics of Solids, 2017, 105, pp.25 - 53. 10.1016/j.jmps.2017.04.016 . hal-01627522

\section{HAL Id: hal-01627522}

https://hal-polytechnique.archives-ouvertes.fr/hal-01627522

Submitted on 21 Dec 2017

HAL is a multi-disciplinary open access archive for the deposit and dissemination of scientific research documents, whether they are published or not. The documents may come from teaching and research institutions in France or abroad, or from public or private research centers.
L'archive ouverte pluridisciplinaire HAL, est destinée au dépôt et à la diffusion de documents scientifiques de niveau recherche, publiés ou non, émanant des établissements d'enseignement et de recherche français ou étrangers, des laboratoires publics ou privés. 


\title{
Effective response of classical, auxetic and chiral magnetoelastic materials by use of a new variational principle
}

\author{
K. Danas \\ LMS, C.N.R.S., École Polytechnique, Université Paris-Saclay, 91128 Palaiseau, France
}

\begin{abstract}
This work provides a rigorous analysis of the effective response, i.e., average magnetization and magnetostriction, of magnetoelastic composites that are subjected to overall magnetic and mechanical loads. It clarifies the differences between a coupled magnetomechanical analysis in which one applies a Eulerian (current) magnetic field and an electroactive one where the Lagrangian (reference) electric field is usually applied. For this, we propose an augmented vector potential variational formulation to carry out numerical periodic homogenization studies of magnetoelastic solids at finite strains and magnetic fields. We show that the developed variational principle can be used for bottom-up design of microstructures with desired magnetomechanical coupling by properly canceling out the macro-geometry and specimen shape effects. To achieve that we properly treat the average Maxwell stresses arising from the medium surrounding the magnetoelastic representative volume element (RVE) while at the same time we impose a uniform average Eulerian-and not Lagrangian-magnetic field. The developed variational principle is then used to study a large number of ideal as well as more realistic two-dimensional microstructures. We study the effect of particle volume fraction, particle distribution and particle shape and orientation upon the effective magnetoelastic response at finite strains. We consider also unstructured isotropic microstructures based on random adsorption algorithms and we carry out a convergence study of the representativity of the proposed unit cells. Finally, three-phase two-dimensional auxetic microstructures are analyzed. The first consists of a periodic distribution of voids and particle chains in a polymer matrix, while the second takes advantage of particle shape and chirality to produce negative and positive swelling by proper change of the chirality and the applied magnetic field.
\end{abstract}

Key words: Magnetorheological elastomers, Magnetoelasticity, Finite strain, Finite elasticity, Homogenization, Magnetostriction, Auxetic, Chirality

\section{Introduction}

In recent years, there has been an increasing interest in the experimental, theoretical and numerical study of magnetoelastic and electroelastic materials that can achieve large strains under application of magnetic or electric fields. In the context of magnetoelasticity, this has been possible by the fabrication of composite materials made of a soft matrix (such as gels, polymers and elastomers) and magnetically soft but mechanically stiff particles. This class of materials has been termed magnetorheological elastomers (MREs) or magnetorheological polymers (MRPs) (Ginder et al., 1999; Martin et al., 2006; Diguet et al., 2010; Danas et al., 2012). In all those experimental studies the authors considered a specimen of given dimensions which is subjected to uniform Eulerian magnetic fields. One of the goals in those studies was the magnetomechanical characterization of the MRE, i.e., the constitutive description of the composite. An important feature raised by some of these authors (Brown, 1966; Martin et al., 2006; Diguet et al., 2010)

Email addresses: konstantinos.danas@polytechnique.edu (K. Danas) 
was the effect of specimen shape in the obtained magnetostriction ${ }^{1}$ of the composite.

The main reason for this specimen shape effect can be directly attributed to the experimental setup and corresponding boundary conditions. By observation of Fig. 1a, usually the MRE specimen is placed at a given distance between two magnetic poles, while the background magnetic field is created far from it. This has two major consequences. First, the Maxwell magnetic stresses outside the studied material or representative volume element (RVE) are not zero and thus stress jumps along boundaries and interfaces have to be properly addressed. Second, the background magnetic field does not change with the specimen deformation and thus is Eulerian in nature. Moreover, by referring to the early works of Eshelby (1957) and Brown (1966) (see also recent work by Liu et al. (2006)), we know that when boundary conditions are applied far from an inclusion (in this case the specimen can be considered as the inclusion), the deformation of the inclusion itself depends upon its shape and its orientation with respect to the applied fields. Evidently, the measured specimen deformations are functions of the specimen shapes as well as of the material properties of the specimen itself. It is thus not trivial to decouple the deformation due to the specimen shape and the material properties, while taking into account the jump in Maxwell stresses.

a)

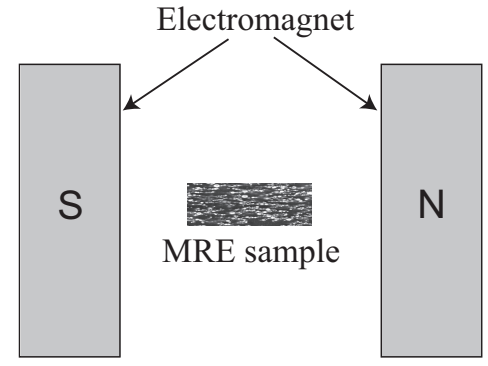

b)



Figure 1: Standard experimental setups for the study of (a) magnetorheological elastomers and (b) electroactive polymers.

In turn, in the context of electroelasticity, which is described by the same set of equations, very often experiments are carried out by spraying very soft (or liquid) electrodes on the top and bottom surfaces of a thin polymer layer, i.e., the electric field is applied directly on the boundary of the solid (similar to mechanical testing), as shown in the sketch of Fig. 1b. The electric field then follows the deformation of the material and hence is Lagrangian in nature, while the overall stresses (Maxwell and mechanical) are identically zero outside the specimen (except near corners). Moreover, since the electric field is applied directly at the boundary of the solid, the specimen shape is irrelevant but the attractive forces of the electrodes contribute now to the deformation of the polymer.

Therefore, a question arising from the above discussion is simply what is the proper way to analyze the effective or homogenized response of magnetoelastic composites free of macroscopic specimen effects. And more specifically, how one could decouple the RVE response from macroscopic specimen/geometry effects and impose a Eulerian magnetic field, which implies a non-proportional loading path in the space of the deformation gradient and the Lagrangian magnetic fields. The response to that question is one of the principal goals of the present study.

With regard to the magnetoelastic problem, a number of studies - theoretical and numerical-were devoted to the constitutive description of MREs (see theoretical works by Dorfmann and Ogden (2003), Kankanala and Triantafyllidis (2004), Ericksen (2006) and more recently a rather complete formulation by Liu (2014)). From the theoretical point of view, nonlinear homogenization models have been developed (Ponte Castañeda and Galipeau, 2011) based on a linear comparison composite method and a par-

\footnotetext{
${ }^{1}$ In the present study, we specify that the notation magnetostriction refers to the overall deformation of the composite (one can also call it extrinsic magnetostriction) and not the deformation of the particles themselves (which can be called as intrinsic magnetostriction).
} 
tial decoupling approach. In this study it was shown that the overall magnetostriction of the composite is due to particle interactions, which can be enhanced by considering particles with elliptical shapes (Galipeau and Ponte Castañeda, 2013; Lefèvre and Lopez-Pamies, 2015). A homogenization model in the context of magnetorheological laminates and their stability has also been developped by Rudykh and Bertoldi (2013).

In parallel, numerical homogenization of simple microstructures was carried out in two-dimensional and three-dimensional unit cells (Javili et al., 2013; Galipeau et al., 2014; Miehe et al., 2016). In those studies, the results were obtained by using a scalar potential formulation which was presented in the context of electromechanical problems by Landis (2002) (but see also recent numerical work by Henann et al. (2013)). In more recent studies, Kästner and co-workers (Spieler et al., 2013, 2014) have developed a vector potential staggered algorithm at small and finite strains in two-dimensions to study MREs. In a recent work, following the boundary conditions proposed by Galipeau et al. (2014), Metsch et al. (2016) have carried out homogenization calculations to obtain estimates for unstructured (e.g., isotropic) and structured (particle-chain like) MREs. In that work, they have assumed a rectangular macroscopic specimen geometry and then imposed Maxwell stresses on the boundaries of the unit cell as those are obtained by imposing certain continuity of the tractions with the surrounding air. Nonetheless, as they state in their study such a procedure neglects perturbations of the macroscopic magnetic fields and inherently involves a given specimen geometry.

To improve on this, in a more recent study, Kalina et al. (2016) have modeled directly the specimen, the surrounding air and the microstructure at the same scale. While this study has led to satisfactory qualitative agreement with experiments, it did not resolve the different length scales as one goes from specimen to microstructure, since that would require an untractable mesh size. Along this effort, Keip and Rambausek (2015) proposed a two-scale finite element approach in order to solve simultaneously the magnetomechanical boundary value problem (see Fig. 1a) and the microstructural problem by properly resolving the separation of the very different length scales. While this last approach is the more complete one, to our personal point of view, in terms of understanding all the features of the problem, it still remains numerically demanding, especially if complex unit cells with large number of particles are considered. Moreover, in all these approaches, it is very hard to decouple from the estimated response the relative effect of the specimen geometry and that of the microstructure.

In an effort to provide a rigorous mathematical tool for design of microstructures with desired magnetomechanical coupling that are free of specimen shape effects, we propose an augmented vector potential variational formulation, which derives directly from the variational principle of Dorfmann and Ogden (2003) by subtracting two terms. The first term serves to impose the average Maxwell stress continuity at the RVE scale arising due to the medium surrounding the magnetoelastic representative volume element and therefore neutralize any specimen/shape effects. The second term allows us to apply the average Eulerian magnetic field - which is the natural one to apply in an experiment such as the one sketched in Fig. 1a - instead of the Lagrangian one (see Fig. 1b). The proposed augmented variational principle is shown in the Appendix A to be exactly equivalent to the variational principle of Kankanala and Triantafyllidis (2004) and James and Kinderlehrer (1993) (see also in the same spirit the work of De Simone (1993)) as well as that of Ericksen (2006) (in the purely magnetic case).

The developed homogenization tool is then used to study a large number of two-dimensional microstructures free of specimen shape effects. We study extensively the effect of particle volume fraction, particle distribution and particle shape and orientation upon the effective magnetoelastic response at finite strains. We also consider random isotropic microstructures based on random adsorption algorithms and we carry out a convergence study of the representativity of the proposed unit cells. Two new more "exotic" microstructures that lead to auxetic responses are also proposed. The first one refers to an auxetic MRE and is inspired by the works of Michel et al. (2007), Bertoldi et al. (2008), Bertoldi et al. (2010) and more recently Tipton et al. (2012) in the context of magnetomechanical loads. This type of unit cell has been shown to give an in-plane negative Poisson ratio response when under compression at a macrostructural level. The second microstructure, which is inspired by relevant work of Prall and Lakes (1997) and Spadoni et al. (2009), leads to magnetically-driven negative and positive swelling by proper use of geometrical chirality of the microstructure. Both of these microstructures are certainly realizable at a laboratory level and such a work is underway. It should also be mentioned that the proposed variational principle can be readily applied 
in three-dimensions, but here we focus on two-dimensional results.

Following this introduction, we define the kinematics and magnetostatics as well as the local constitutive laws in Section 2. Next, in Section 3, we describe the homogenization problem, which is appropriately augmented in Section 4. We then proceed to the presentation of two-dimensional results for several microstructures in Sections 5 and 6. We conclude the study in Section 7. Finally, in Appendix A, we compare the proposed augmented variational principle with the ones of Kankanala and Triantafyllidis (2004) and James and Kinderlehrer (1993) and propose other equivalent forms that could serve for a range of boundary value problems beyond homogenization. We finally discuss in Section B details on the application of periodic boundary conditions in two-dimensions.

\section{Magneto-elastostatics and local constitutive behavior}

We consider a magnetoelastic deformable solid that occupies a region $\mathcal{V}_{0}$ (and $\left.\mathcal{V}\right)$ with boundary $\partial \mathcal{V}_{0}$ (and $\partial \mathcal{V}$ ) of outward normal $\mathcal{N}$ (and $\mathbf{n}$ ) in the undeformed stress-free (current) configuration. Material points in the solid are identified by their initial position vector $\mathbf{X}$ in the undeformed configuration $\mathcal{V}_{0}$, while the current position vector of the same point in the deformed configuration $\mathcal{V}$ is given by $\mathbf{x}=\boldsymbol{\chi}(\mathbf{X})$. Motivated by the usual physical arguments, the mapping $\chi$ is required to be continuous and one-to-one on $\mathcal{V}_{0}$. In addition, we assume that $\chi$ is twice continuously differentiable, except possibly on existing interfaces (e.g., due to the presence of different phases) inside the material. The deformation gradient is then denoted by $\mathbf{F}=\operatorname{Grad} \boldsymbol{\chi}$ and its determinant by at $J=\operatorname{det} \mathbf{F}>0$. Here, Grad denotes the gradient operator with respect to $\mathbf{X}$ in the reference configuration. In addition, the reference density of the solid $\rho_{0}$ is related to the current density $\rho$ by $\rho_{0}=\rho J$. Time dependence in not considered here.

In pure magnetics and in the absence of deformation $(\mathbf{F}=\mathbf{I})$, the current magnetic field $\mathbf{b}$ is divergencefree, the $h$-field $\mathbf{h}$ is curl-free and the magnetization per unit current volume is defined by

$$
\mathbf{b}=\mu_{0}(\mathbf{h}+\mathbf{m}) \quad \text { in } \quad \mathcal{V}
$$

where $\mu_{0}$ is the magnetic permeability in vacuum (to be specified later) and $\mathcal{V}$ is the volume in the current configuration. This equation is used to identify one out of the three vector fields when one vector field is used as an independent variable and the other one is given by a constitutive equations, e.g., $\mathbf{h}=f(\mathbf{b})$. In general, and in purely mathematical terms, one could choose any of the above as an independent variable. Note, however, that $\mathbf{b}$ and $\mathbf{h}$ are a priori Eulerian quantities that need to satisfy differential constraints and boundary conditions, whereas the magnetization $\mathbf{m}$, which is also a Eulerian quantity by definition through equation (2.1), does not need to satisfy any differential constraints or interface conditions.

At large strains, the fields $\mathbf{b}$ and $\mathbf{h}$ can be pulled back from $\mathcal{V}$ to $\mathcal{V}_{0}$ to their Lagrangian forms, denoted by $\mathbf{B}$ and $\mathbf{H}$, respectively, such that (Dorfmann and Ogden, 2003; Bustamante et al., 2008)

$$
\mathbf{B}=J \mathbf{F}^{-1} \mathbf{b}, \quad \text { and } \quad \mathbf{H}=\mathbf{F}^{T} \mathbf{h} .
$$

No attempt is done to provide any Lagrangian form for $\mathbf{m}$ since that would be non unique and is of no use in the subsequent analysis. Moreover, as has been extensively discussed in the literature (see for instance Dorfmann and Ogden (2005)), equation (2.1) is not form invariant under transformations, which is a manifestation of the non-unique definition of $\mathbf{m}$. The Lagrangian $\mathbf{B}$ is also divergence-free and $\mathbf{H}$ is curl-free. Those last properties allow us to introduce the magnetic vector potential A, i.e.,

$$
\operatorname{Div} \mathbf{B}=0 \quad \Leftrightarrow \quad \mathbf{B}=\mathrm{Curl} \mathbf{A} \quad \text { in } \quad \mathcal{V}_{0}, \quad \text { and } \quad \llbracket \mathbf{A} \rrbracket \times \mathcal{N}=0 \quad \text { in } \partial \mathcal{V}_{0} .
$$

Finally, following the works of Dorfmann and Ogden (2003), Kankanala and Triantafyllidis (2004) and Bustamante et al. (2008), we define the total Cauchy stress tensor $\boldsymbol{\sigma}$ and the total (first) Piola-Kirchhoff $\mathbf{S}$ via the relation

$$
\mathbf{S}=J \boldsymbol{\sigma} \mathbf{F}^{-T}, \quad \boldsymbol{\sigma}=\boldsymbol{\sigma}^{\text {mech }}+\boldsymbol{\sigma}^{\text {maxw }}, \quad \text { with } \quad \boldsymbol{\sigma}^{\text {maxw }}=\mathbf{h} \otimes \mathbf{b}-\frac{\mu_{0}}{2}\left(|\mathbf{h}|^{2}-|\mathbf{m}|^{2}\right) \mathbf{I} .
$$


where |.| denotes the standard Euclidean norm of a vector, $\mathbf{I}$ is the second order identity tensor, whereas $\boldsymbol{\sigma}^{\text {mech }}$ and $\boldsymbol{\sigma}^{\text {maxw }}$ denote the mechanical and Maxwell parts, respectively. This of course is a convenient mathematical description as for instance it allows for an easier analysis of the present homogenization problem, but is not unique (see for instance Kankanala and Triantafyllidis (2004)). Again, both $\mathbf{S}$ and $\boldsymbol{\sigma}$ are divergence free fields.

Next, we define constitutive laws for the coupled magnetomechanical response of the materials under study. The interest in this study is the modeling of composite materials which can be either magnetoelastic or purely elastic. In this regard, it is convenient to characterize their constitutive behaviors in a Lagrangian formulation by free energies $W(\mathbf{X}, \mathbf{F}, \mathbf{B})$. These functions are suitably amended in order to include the contribution of the Maxwell stress and read (Dorfmann and Ogden, 2005)

$$
W(\mathbf{X}, \mathbf{F}, \mathbf{B})=\rho_{0} \Phi(\mathbf{X}, \mathbf{F}, \mathbf{B})+\frac{1}{2 \mu_{0} J} \mathbf{F} \mathbf{B} \cdot \mathbf{F} \mathbf{B}
$$

where $\Phi(\mathbf{X}, \mathbf{F}, \mathbf{B})$ is a specific free-energy density to be defined below, while the second term in the above equation is considered so that the total first Piola-Kirchhoff stress $\mathbf{S}$ and the Lagrangian $h$-field $\mathbf{H}$ are simply given by

$$
\mathbf{S}=\frac{\partial W}{\partial \mathbf{F}}(\mathbf{X}, \mathbf{F}, \mathbf{B}), \quad \mathbf{H}=\frac{\partial W}{\partial \mathbf{B}}(\mathbf{X}, \mathbf{F}, \mathbf{B}) .
$$

Next, we consider that the composite material comprises $r=1, \ldots, N$ (magneto)elastic phases occupying a subdomain $\mathcal{V}_{0}^{(r)}$ in the reference configuration so that the local potential $W$ is re-written simply as

$$
W(\mathbf{X}, \mathbf{F}, \mathbf{B})=\sum_{r=1}^{N} \Theta_{0}^{(r)}(\mathbf{X}) W^{(r)}(\mathbf{F}, \mathbf{B}) .
$$

In this expression, $\Theta_{0}^{(r)}(\mathbf{X})$ denote characteristic functions that describe the distribution of phase $r$ and take values $\Theta_{0}^{(r)}(\mathbf{X})=1$ if $\mathbf{X} \in \mathcal{V}_{0}^{(r)}$ and zero otherwise. Each phase then is described by an energy function $W^{(r)}(\mathbf{F}, \mathbf{B})$ or equivalently $w^{(r)}(\mathbf{F}, \mathbf{b})$, which takes the general form

$$
\begin{aligned}
W^{(r)}(\mathbf{F}, \mathbf{B}) & =\rho_{0}^{(r)} \Phi^{(r)}(\mathbf{F}, \mathbf{B})+\frac{1}{2 \mu_{0} J} \mathbf{F} \mathbf{B} \cdot \mathbf{F} \mathbf{B}= \\
& =\rho_{0}^{(r)} \phi^{(r)}(\mathbf{F}, \mathbf{b})+\frac{J}{2 \mu_{0}} \mathbf{b} \cdot \mathbf{b}=w^{(r)}(\mathbf{F}, \mathbf{b}) .
\end{aligned}
$$

Here, we have used relation $(2.2)_{1}$ to obtain the property $W^{(r)}(\mathbf{F}, \mathbf{B})=W^{(r)}\left(\mathbf{F}, J^{-1} \mathbf{F}^{-1} \mathbf{b}\right)=w^{(r)}(\mathbf{F}, \mathbf{b})$. In the case of periodic composites these functions are periodic and are fully determined by the unit cell occupying a domain $\mathcal{V}_{0}^{\#}$ such that $\Theta_{0}^{(r)}(\mathbf{X})=\sum_{q_{1}, q_{2}, q_{3} \in \mathbb{Z}} \Theta_{0}^{(r)}\left(\mathbf{X}+q_{1} \mathbf{L}^{1}+q_{2} \mathbf{L}^{2}+q_{3} \mathbf{L}^{3}\right.$ ) and $\mathbf{X} \in \mathcal{V}_{0}^{\#}$ (Michel et al., 1999). Here, the unit cell is assumed to be a parallelepiped defined via the lattice vectors $\mathbf{L}^{i}(i=1,2,3)$ in the reference configuration. In any case, the smallest unit cell $\mathcal{V}_{0}^{\#}$ suffices in the examples discussed in this work.

\subsection{Choice of energy functions}

In this section, we propose simple but sufficiently rich energy density functions to describe the constitutive response of the constituents comprising the magnetoelastic composite materials under study in this work. For this, it suffices to define forms for the specific energy density $\Phi^{(r)}(\mathbf{F}, \mathbf{B})$ as introduced in equation (2.7).

A number of phenomenological energy functions - isotropic and anisotropic - have been proposed in the literature (Bustamante, 2010; Ponte Castañeda and Galipeau, 2011; Danas et al., 2012). In this study, we focus only on simple isotropic energy functions with saturation of the magnetization. In addition, in all cases simulated in this work, we will consider magnetic particles that are almost rigid mechanically but magnetically soft. This allows for use of simple magnetoelastic functions for the constituents. However, the 
homogenization framework that is developed in the following sections is general and can deal with arbitrary complexity of the magnetoelastic response of the constituents.

In this regard, for a homogeneous magnetoelastic solid, we consider the following energy density

$$
\begin{aligned}
\rho_{0}^{(r)} \Phi^{(r)}(\mathbf{F}, \mathbf{B}) & =\rho_{0}^{(r)} \Phi_{m e c h}^{(r)}(\mathbf{F})+\rho_{0}^{(r)} \Phi_{m a g}^{(r)}(\mathbf{F}, \mathbf{B})= \\
& =\rho_{0}^{(r)} \phi_{m e c h}^{(r)}(\mathbf{F})+\rho_{0}^{(r)} \phi_{m a g}^{(r)}(\mathbf{F}, \mathbf{b})=\rho_{0}^{(r)} \phi^{(r)}(\mathbf{F}, \mathbf{b}) .
\end{aligned}
$$

Use has been made of again of relation $(2.2)_{1}$ to obtain the property $\Phi^{(r)}(\mathbf{F}, \mathbf{B})=\Phi^{(r)}\left(\mathbf{F}, J^{-1} \mathbf{F}^{-1} \mathbf{b}\right)=$ $\phi^{(r)}(\mathbf{F}, \mathbf{b})$, while the term with subscript "mech" in the above equation is a purely mechanical contribution and for the purposes of the present work and without lose of generality, we choose a simple Neo-Hookean model, i.e.,

$$
\rho_{0}^{(r)} \Phi_{m e c h}^{(r)}(\mathbf{F})=\frac{G}{2}(\mathbf{F} \cdot \mathbf{F}-3-2 \ln J)+\frac{G^{\prime}}{2}(J-1)^{2},
$$

where $G$ is the shear modulus and $G^{\prime}$ is the Lamé constant associated with the compressible part. In turn, one possibility for the magnetomechanical part is the use of a Langevin model, which reads

$$
\rho_{0}^{(r)} \Phi_{m a g}^{(r)}(\mathbf{F}, \mathbf{B})=\frac{J \mu_{0}\left(m_{s}^{(r)}\right)^{2}}{3 \chi^{(r)}}\left\{\ln \left[\frac{3 \chi^{(r)}}{\mu_{0} m_{s}^{(r)} J} \sqrt{\mathbf{F B} \cdot \mathbf{F B}}\right]-\ln \left[\sinh \left(\frac{3 \chi^{(r)}}{\mu_{0} m_{s}^{(r)} J} \sqrt{\mathbf{F B} \cdot \mathbf{F B}}\right)\right]\right\}
$$

or equivalently in terms of the Eulerian $\mathbf{b}$ as

$$
\rho_{0}^{(r)} \phi_{m a g}^{(r)}(\mathbf{F}, \mathbf{b})=\frac{J \mu_{0}\left(m_{s}^{(r)}\right)^{2}}{3 \chi^{(r)}}\left\{\ln \left[\frac{3 \chi^{(r)}}{\mu_{0} m_{s}^{(r)}}|\mathbf{b}|\right]-\ln \left[\sinh \left(\frac{3 \chi^{(r)}}{\mu_{0} m_{s}^{(r)}}|\mathbf{b}|\right)\right]\right\}
$$

In these two expressions, $|\mathbf{b}|=\sqrt{\mathbf{b} \cdot \mathbf{b}}, \mu_{0}$ is the magnetic permeability in vacuum (to be specified later), $m_{s}^{(r)}$ is the magnetic saturation and $\chi^{(r)}$ is the magnetic susceptibility of phase $r$. There are more than one definitions of magnetic susceptibility in the literature. The one used here is the more recent one in the bibliography whereas the classical one is $\chi_{v}^{(r)}=\chi^{(r)} /\left(1-\chi^{(r)}\right)$. For instance, in the absence of mechanical fields (e.g., rigid solids), the relative permeability $\mu^{(r)}=\left(1+\chi_{v}^{(r)}\right) \mu_{0}=\mu_{0} /\left(1-\chi^{(r)}\right)$ of iron could be in the order of $\chi_{v}^{(r)} \approx 200$ or $\chi^{(r)} \approx 0.995$.

For the sake of generality in our presentation, we mention that in order to model magnetic saturation one could also use different energy densities than that in equation (2.11). One such alternative energy reads

$$
\rho_{0}^{(r)} \phi_{m a g}^{(r)}(\mathbf{F}, \mathbf{b})=-\frac{J \mu_{0}\left(m_{s}^{(r)}\right)^{2}}{\chi^{(r)}} \ln \left[\cosh \left(\frac{\chi^{(r)}}{\mu_{0} m_{s}^{(r)}}|\mathbf{b}|\right)\right] .
$$

This energy is exactly equivalent to the one in (2.11) for small magnetic fields, as will be shown in the following, whereas it leads to a "tanh" response in the $\mathbf{m}-\mathbf{b}$ space. In fact, the energy in (2.12) exhibits a faster growth and reaches the saturation magnetization faster than that in equation (2.11).

The magnetization is then defined by simple derivation of equation (2.11) (the same result could be obtained by proper derivation of (2.10) but is much more tedious) as

$$
\begin{aligned}
\mathbf{m} & =-\frac{\rho_{0}^{(r)}}{J} \frac{\partial \phi^{(r)}}{\partial \mathbf{b}}=-\frac{\rho_{0}^{(r)}}{J} \frac{\partial \phi_{m a g}^{(r)}}{\partial \mathbf{b}}=-\frac{\rho_{0}^{(r)}}{J} \frac{\partial \Phi_{m a g}^{(r)}}{\partial \mathbf{B}} \mathbf{F}^{-T} \\
& =\left(\frac{m_{s}^{(r)}}{|\mathbf{b}|} \operatorname{coth}\left[\frac{3 \chi^{(r)}|\mathbf{b}|}{\mu_{0} m_{s}^{(r)}}\right]-\frac{\mu_{0}\left(m_{s}^{(r)}\right)^{2}}{3 \chi^{(r)}|\mathbf{b}|^{2}}\right) \mathbf{b}
\end{aligned}
$$

or alternatively of equation (2.12) as

$$
\mathbf{m}=-\frac{\rho_{0}^{(r)}}{J} \frac{\partial \phi_{\text {mag }}^{(r)}}{\partial \mathbf{b}}=\frac{m_{s}^{(r)}}{|\mathbf{b}|} \tanh \left[\frac{\chi^{(r)}|\mathbf{b}|}{\mu_{0} m_{s}^{(r)}}\right] \mathbf{b}
$$


so that $\mu_{0} \mathbf{h}=\mathbf{b}-\mu_{0} \mathbf{m}(\mathbf{b})$. The magnetization response corresponding to the two different energy densities (2.11) and (2.12) is shown in Fig. 2 as a function of the magnetic field $\mathbf{b}$. Note at this point that any interpolation between these two energy densities (2.11) and (2.12) can be readily used if required by experimental data.

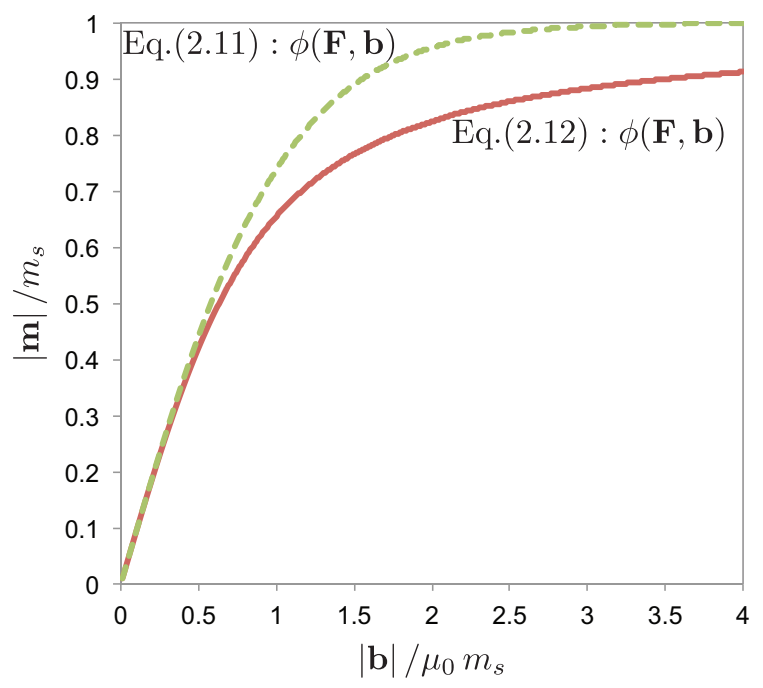

Figure 2: $m-b$ response as obtained by the two different energy densities described in equations (2.11) and (2.12) in the purely magnetic case, i.e., $\mathbf{F}=\mathbf{I}$ and $J=1$, and for a magnetic susceptibility $\chi^{(r)}=\chi=0.995$.

In the limit of small magnetic fields, $\mathbf{B} \rightarrow \mathbf{0}$, one can show that both of the above defined energies $(2.11)$ (or equivalently (2.10)) and (2.12) are the same and become quadratic in $\mathbf{B}$ or $\mathbf{b}$, respectively, such that

$$
\rho_{0}^{(r)} \Phi_{m a g}^{(r)}(\mathbf{F}, \mathbf{B})=-\frac{\chi^{(r)}}{2 \mu_{0} J} \mathbf{F} \mathbf{B} \cdot \mathbf{F} \mathbf{B}=-\frac{J \chi^{(r)}}{2 \mu_{0}} \mathbf{b} \cdot \mathbf{b}=\rho_{0}^{(r)} \phi_{m a g}^{(r)}(\mathbf{F}, \mathbf{b}) .
$$

This last case corresponds to ideal magnetic materials with no saturation. Using equation (2.13), one can easily show that the magnetization in this last case is linear in $\mathbf{b}$ and is given by

$$
\mu_{0} \mathbf{m}=\chi^{(r)} \mathbf{b} \quad \text { or } \quad \mu_{0} \mathbf{h}=\left(1-\chi^{(r)}\right) \mathbf{b} .
$$

The constitutive relations discussed in this section are not of course the only ones that can be used to describe the phenomena observed in experiments (see for instance Danas et al. (2012)) but are representative and serve sufficiently the purpose of this study.

In the following sections, we use mostly the Langevin saturation model given by (2.11), except in Section 5.5, where we compare the Langevin model (2.11) with the "tanh" saturation model given by (2.12). It is noted, however, that the qualitative effective response of the magnetoelastic composites under study is independent of that choice.

\section{Periodic homogenization framework}

In this section, we provide the reader with some basic definitions in the context of homogenization and define the problem at hand. By referring to the experimental setup in Fig. 1, we discuss first the different scales that are present in such magnetoelastic problems, as shown in Fig. 3. Specifically, Figure 3a shows a standard magnetoelastic boundary value problem (BVP), which involves a specimen of arbitrary shape embedded in the surrounding air, i.e., the ether medium lying between the magnetic poles which are usually placed far from the solid. The external field $\mathbf{b}_{0}$ applied in the background (at the macroscale) by the magnets 
interacts with the specimen, which in turn creates a self-field. The sum of these two fields results in an otherwise heterogeneous true magnetic field at the macroscale seen by any material point of the solid. At a given material point and its neighborhood at the macroscale, as depicted by the green box in Fig. 3a, the stress, strain and true magnetic fields are denoted by $\overline{\mathbf{S}}, \overline{\mathbf{F}}, \overline{\mathbf{b}}, \overline{\mathbf{h}}$ and $\overline{\mathbf{m}}$, respectively. Assuming a slowly varying microstructure, these fields correspond to the average fields at the representative volume element (RVE) scale and its immediate neighborhood, as shown in Fig. 3b. One should note also that $\overline{\mathbf{b}}, \overline{\mathbf{h}}$ and $\overline{\mathbf{m}}$ depend on $\mathbf{b}_{0}$, the geometry of the specimen and its relative position to the magnets. While at the macroscale, the field $\overline{\mathbf{b}}$ is the true field at a given material point, at the RVE scale (i.e., microscale) it is the average (background) field. In turn, the true Eulerian magnetic field $\mathbf{b}$ at the RVE scale is the sum of the average magnetic field $\overline{\mathbf{b}}$ and the perturbation field $\widetilde{\mathbf{b}}$ (with zero average), as will be discussed in detail in the following.



d) Periodic RVE

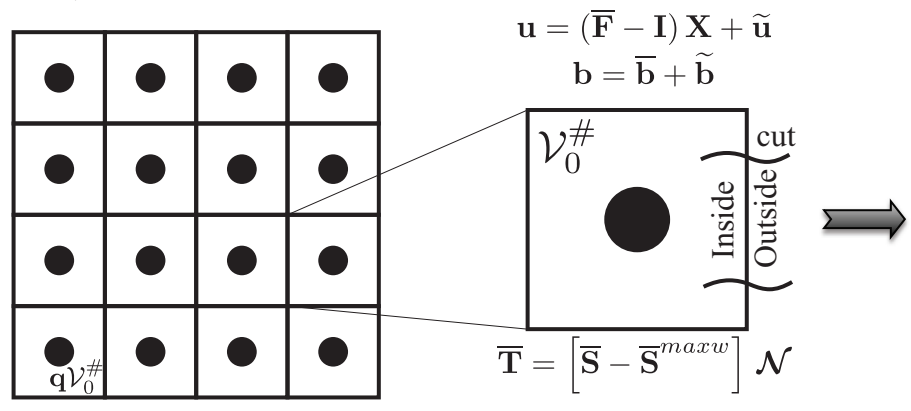

e) Periodic unit cell

$\mathbf{u}=(\overline{\mathbf{F}}-\mathbf{I}) \mathbf{X}+\widetilde{\mathbf{u}}$ f) Boundary Cut

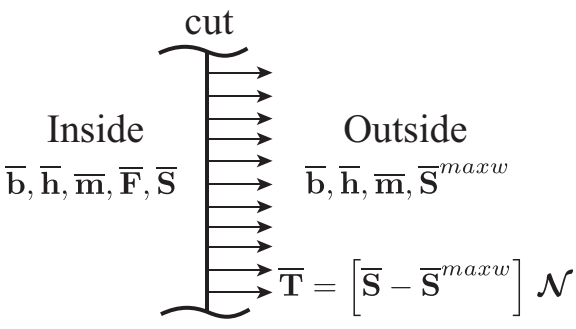

Figure 3: Problem setting defining the separation of scales as one goes from the standard magnetomechanical boundary value problem to the local homogenization problem and the corresponding periodic one.

Following well-known concepts in homogenization, we assume complete separation of length scales as one goes from the macroscale (a material point denoted by the green box in Fig. 3a) to the RVE microscale (Figs. 3b-e). Moreover, one may further simplify the RVE of Fig. 3c as shown for illustration in Fig. 3d. The simplified periodic RVE occupies a volume $\mathbf{q} \mathcal{V}_{0}^{\#}$ and comprises simpler periodic unit cells of volume $\mathcal{V}_{0}^{\#}$, as shown in Fig. 3e. This periodic unit cell, in turn, is subjected to periodic boundary conditions to enforce representativity of the volume element.

As already mentioned in the Introduction, in this work, we aim at carrying out the calculation of the average magnetomechanical response at the scale of the RVE independently of the specimen shape, 
the magnetic and strain gradients or the presence of the surrounding air. By conrast, the macroscale effects can only be resolved at the upper scale via direct simulation of the geometry (see relavant work of Keip and Rambausek (2015)). In order to achieve the decoupling of the macroscale and the microscale, one needs to properly extract the macroscale effects and analyze only the given RVE subjected to average stress, strain and magnetic fields. This will allow then to understand the magnetomechanical coupling solely due to the microstructure. In view of this, we first observe that an RVE, which describes the effective response at a single material point (green box in Fig. 3a) far from boundaries, is surrounded by other neighboring RVEs (and not the air), as shown in Fig. 3b, and thus the average Maxwell stress, denoted by $\overline{\mathbf{S}}$ maxw , exerted upon this RVE is a function of the average deformation gradient $\overline{\mathbf{F}}$ and the average magnetic fields $\overline{\mathbf{b}}, \overline{\mathbf{h}}$ and $\overline{\mathbf{m}}$ at the immediate neighborhood of this RVE. It is stressed here that the magnetization outside a given RVE or equivalently unit cell shall not be considered zero as if air was outside but should be nonzero as if a neighboring RVE is outside with (almost) the same properties, assuming slowly varying material properties at the macroscale, as illustrated in Fig. 3b. By definition, then, the average "mechanical" traction $\overline{\mathbf{T}}$ on the boundary of the RVE or periodic unit cell is the difference between the average total stress $\overline{\mathbf{S}}$ inside the RVE and the average Maxwell stress $\overline{\mathbf{S}}^{\operatorname{maxw}}$ outside the RVE, as graphically illustrated in Fig. 3f. Extracting this average Maxwell stress during the periodic homogenization procedure is a non-trivial operation and is discussed in Section 4.

Following the description of the problem at hand, we define the volume average deformation gradient and magnetic field in $\mathbf{q} \mathcal{V}_{0}^{\#}\left(\right.$ where $\left.\mathbf{q}=\left(q_{1}, q_{2}, q_{3}\right)\right)$ unit cells as

$$
\overline{\mathbf{F}}=\frac{1}{\left|\mathbf{q} \mathcal{V}_{0}^{\#}\right|} \int_{\left|\mathbf{q} \mathcal{V}_{0}^{\#}\right|} \mathbf{F}(\mathbf{X}) \mathrm{d} V, \quad \overline{\mathbf{B}}=\frac{1}{\left|\mathbf{q} \mathcal{V}_{0}^{\#}\right|} \int_{\left|\mathbf{q} \mathcal{V}_{0}^{\#}\right|} \mathbf{B}(\mathbf{X}) \mathrm{d} V .
$$

The definition of periodicity in two and three dimensions implies that the displacement field and magnetic vector field are periodic about an average strain and magnetic field, respectively, such that

$$
\begin{aligned}
& \mathbf{u}(\mathbf{X})=(\overline{\mathbf{F}}-\mathbf{I}) \mathbf{X}+\widetilde{\mathbf{u}}(\mathbf{X}), \quad \mathbf{A}(\mathbf{X})=\overline{\mathbf{A}}(\mathbf{X})+\widetilde{\mathbf{A}}(\mathbf{X}) \\
& 3 D: \bar{A}_{i}=\frac{1}{2} \varepsilon_{i j k} \bar{B}_{j} X_{k} \quad(i=1,2,3), \quad 2 D: \bar{A}_{3}=\varepsilon_{\beta \gamma} \bar{B}_{\beta} X_{\gamma},
\end{aligned}
$$

where $\varepsilon_{i j k}$ and $\varepsilon_{\beta \gamma}$ are the permutation symbols, in three and two dimensions, respectively, $\mathcal{N}$ is the reference unit normal of the boundary in the reference configuration and $\widetilde{\mathbf{u}}$ and $\widetilde{\mathbf{A}}$ are periodic fields with zero mean value. Repeated indices denote standard summation in the above expression with $i, j, k=1,2,3(3 \mathrm{D})$ and $\beta, \gamma=1,2$ (2D). Application of the above-described periodic boundary conditions is detailed in Appendix B.

Use of the above periodic boundary conditions and the fact that $\mathbf{B}$ is divergence free leads to the corresponding average eulerian magnetic field $\mathbf{b}$ in the current configuration

$$
\overline{\mathbf{b}}=\frac{1}{\mid \mathbf{q} \mathcal{V}^{\# \mid}} \int_{|\mathbf{q} \mathcal{V} \#|} \mathbf{b}(\mathbf{x}) \mathrm{d} v=\bar{J}^{-1} \overline{\mathbf{F}} \overline{\mathbf{B}}, \quad \mathcal{V}^{\#}=\bar{J} \mathcal{V}_{0}^{\#}
$$

Moreover, the periodic boundary conditions (3.2) also imply that the magnetic fields can be decomposed to an average magnetic field $\overline{\mathbf{b}}$ and a perturbation magnetic field (with zero average) $\widetilde{\mathbf{b}}$, such that the true magnetic field $\mathbf{b}$ becomes

$$
\mathbf{b}=\overline{\mathbf{b}}+\widetilde{\mathbf{b}}, \quad \text { or } \quad \mathbf{B}=\overline{\mathbf{B}}+\widetilde{\mathbf{B}}
$$

This last relation will be used explicitly in Appendix A to propose alternative equivalent variational formulations.

Using the Hill-Mandel Lemma (see for instance Javili et al. (2013)) and the above defined periodicity conditions, the homogenization problem is defined formally via the optimization problem

$$
\mathcal{P}_{0}(\overline{\mathbf{F}}, \overline{\mathbf{B}})=\widetilde{W}(\overline{\mathbf{F}}, \overline{\mathbf{B}})=\min _{\mathbf{u} \in \mathcal{K}(\overline{\mathbf{F}})} \min _{\mathbf{A} \in \mathcal{A}(\overline{\mathbf{B}})} \frac{1}{\left|\mathbf{q} \mathcal{V}_{0}^{\#}\right|} \int_{\left|\mathbf{q} \mathcal{V}_{0}^{\#}\right|} W(\mathbf{F}, \mathbf{B}) \mathrm{d} V
$$


where $\mathcal{K}$ and $\mathcal{A}$ are the sets of admissible fields for $\mathbf{u}$ and $\mathbf{A}$ denoted by

$$
\mathcal{K}(\overline{\mathbf{F}})=\left\{\mathbf{F}: \exists \mathbf{x}=\chi(\mathbf{X}) \text { with } \mathbf{F}=\mathbf{I}+\operatorname{Grad} \mathbf{u}, J>0, \mathbf{u}=(\overline{\mathbf{F}}-\mathbf{I}) \mathbf{X}+\widetilde{\mathbf{u}}, \widetilde{\mathbf{u}} \text { periodic, in } \mathbf{q} \mathcal{V}_{0}^{\#}\right\}
$$

and

$$
\mathcal{A}(\overline{\mathbf{B}})=\left\{\mathbf{B}: \exists \mathbf{x}=\chi(\mathbf{X}) \text { with } \mathbf{B}=\operatorname{Curl} \mathbf{A}, \mathbf{A}=\overline{\mathbf{A}}+\widetilde{\mathbf{A}}, \widetilde{\mathbf{A}} \text { periodic, } \bar{A}_{i}=\frac{1}{2} \varepsilon_{i j k} \bar{B}_{j} X_{k} \text { in } \mathbf{q} \mathcal{V}_{0}^{\#}\right\},
$$

respectively. It can be easily shown then (Ponte Castañeda and Galipeau, 2011; Javili et al., 2013; Lopez-Pamies, 2014 ) that the macroscopic stress $\overline{\mathbf{S}}$ and $h$-field $\overline{\mathbf{H}}$ satisfy the effective (homogenized) constitutive relations

$$
\overline{\mathbf{S}}=\frac{\partial \widetilde{W}}{\partial \overline{\mathbf{F}}}(\overline{\mathbf{F}}, \overline{\mathbf{B}})=\bar{J} \overline{\boldsymbol{\sigma}} \overline{\mathbf{F}}^{-T}, \quad \overline{\mathbf{H}}=\frac{\partial \widetilde{W}}{\partial \overline{\mathbf{B}}}(\overline{\mathbf{F}}, \overline{\mathbf{B}})=\overline{\mathbf{F}}^{T} \overline{\mathbf{h}},
$$

with $\overline{\boldsymbol{\sigma}}$ and $\overline{\mathbf{h}}$ denoting the corresponding average Cauchy stress and eulerian $h$-field, respectively.

It is very important to note at this point that the above macroscopic stress $\overline{\mathbf{S}}$ (or $\overline{\boldsymbol{\sigma}}$ ) includes both mechanical and Maxwell stress contributions. To put this in a more explicit form, it is helpful to consider energy functions of the general type proposed in equation (2.5). In this case, it is straightforward to show that the homogenized energy $\widetilde{W}$ becomes

$$
\begin{aligned}
\widetilde{W}(\overline{\mathbf{F}}, \overline{\mathbf{B}}) & =\bar{\rho}_{0} \widetilde{\Phi}(\overline{\mathbf{F}}, \overline{\mathbf{B}})+\frac{1}{2 \mu_{0} \bar{J}} \overline{\mathbf{F}} \overline{\mathbf{B}} \cdot \overline{\mathbf{F}} \overline{\mathbf{B}} \\
& =\bar{\rho}_{0} \widetilde{\phi}(\overline{\mathbf{F}}, \overline{\mathbf{b}})+\frac{\bar{J}}{2 \mu_{0}} \overline{\mathbf{b}} \cdot \overline{\mathbf{b}}=\widetilde{w}(\overline{\mathbf{F}}, \overline{\mathbf{b}}),
\end{aligned}
$$

where it is noted that $\overline{\mathbf{b}}=\overline{\mathbf{b}}(\overline{\mathbf{F}}, \overline{\mathbf{B}})$ and the notation $\widetilde{W}(\overline{\mathbf{F}}, \overline{\mathbf{B}})=\widetilde{W}\left(\overline{\mathbf{F}}, J^{-1} \overline{\mathbf{F}}^{-1} \overline{\mathbf{b}}\right)=\widetilde{w}(\overline{\mathbf{F}}, \overline{\mathbf{b}})$ is used. Next, use of definitions (3.8) and by direct derivation of the general form of the energy function $\widetilde{W}$ in equation (3.9), one gets the Lagrangian forms of the total stress and $h$-field,

$$
\overline{\mathbf{S}}=\bar{\rho}_{0} \frac{\partial \widetilde{\Phi}}{\partial \overline{\mathbf{F}}}+\frac{1}{\mu_{0} \bar{J}} \overline{\mathbf{B}} \otimes(\overline{\mathbf{F}} \overline{\mathbf{B}})-\frac{1}{2 \mu_{0} \bar{J}}(\overline{\mathbf{F}} \overline{\mathbf{B}} \cdot \overline{\mathbf{F}} \overline{\mathbf{B}}) \overline{\mathbf{F}}^{-T}, \quad \overline{\mathbf{H}}=\bar{\rho}_{0} \frac{\partial \widetilde{\Phi}}{\partial \overline{\mathbf{B}}}+\frac{1}{\mu_{0} \bar{J}} \overline{\mathbf{F}}^{T}(\overline{\mathbf{F}} \overline{\mathbf{B}}) .
$$

In turn, by use of definition (2.13), one can easily get that the average Eulerian magnetization $\overline{\mathbf{m}}$ by

$$
\overline{\mathbf{m}}=-\frac{\bar{\rho}_{0}}{\bar{J}} \frac{\partial \widetilde{\phi}}{\partial \overline{\mathbf{b}}}=-\bar{\rho}_{0} \overline{\mathbf{F}}^{-T} \frac{\partial \widetilde{\Phi}}{\partial \overline{\mathbf{B}}} .
$$

Combining (3.3) and (3.11) together with the second part of equation $(3.10)_{2}$, one gets

$$
\overline{\mathbf{H}}=-\overline{\mathbf{F}}^{T} \overline{\mathbf{m}}+\frac{1}{\mu_{0}} \overline{\mathbf{F}}^{T} \overline{\mathbf{b}}=\overline{\mathbf{F}}^{T} \overline{\mathbf{h}}, \quad \Leftrightarrow \quad \overline{\mathbf{b}}=\mu_{0}(\overline{\mathbf{h}}+\overline{\mathbf{m}}) .
$$

By contrast, in the stress term of equation (3.10), it is not clear which part is the mechanical and which one is the Maxwell stress, since the term $\partial \widetilde{\Phi} / \partial \overline{\mathbf{F}}$ contains magnetic terms due to the dependence of $\Phi$ on $\overline{\mathbf{B}}$. Therefore it is convenient to resort to the corresponding Eulerian measures. After some algebra, and taking into account the fact that the Eulerian part of the magnetic field $\overline{\mathbf{b}}$ depends on $\overline{\mathbf{F}}$ (c.f., equation (3.3)) leading to the extra cross-term $(\partial \widetilde{w} / \partial \overline{\mathbf{b}})(\partial \overline{\mathbf{b}} / \partial \overline{\mathbf{F}})$, the average Cauchy stress $\overline{\boldsymbol{\sigma}}$ can be written as

$$
\overline{\boldsymbol{\sigma}}=\left.\frac{\partial \widetilde{w}}{\partial \overline{\mathbf{F}}}\right|_{\overline{\mathbf{b}}} \overline{\mathbf{F}}^{T}+\left(\frac{\partial \widetilde{w}}{\partial \overline{\mathbf{b}}} \cdot \frac{\partial \overline{\mathbf{b}}}{\partial \overline{\mathbf{F}}}\right) \overline{\mathbf{F}}^{T}=\overline{\boldsymbol{\sigma}}^{\text {mech }}+\overline{\boldsymbol{\sigma}}^{\text {maxw }}, \quad \text { with } \quad \overline{\mathbf{S}}=\bar{J} \overline{\boldsymbol{\sigma}} \overline{\mathbf{F}}^{-T},
$$

so that the average mechanical Cauchy stress is

$$
\overline{\boldsymbol{\sigma}}^{\text {mech }}=\left.\frac{\partial \widetilde{w}}{\partial \overline{\mathbf{F}}}\right|_{\overline{\mathbf{b}}} \overline{\mathbf{F}}^{T}=\left.\frac{\bar{\rho}_{0}}{\bar{J}} \frac{\partial \widetilde{\phi}}{\partial \overline{\mathbf{F}}}\right|_{\overline{\mathbf{b}}} \overline{\mathbf{F}}^{T}, \quad \text { with } \quad \overline{\mathbf{S}}^{\text {mech }}=\bar{J} \overline{\boldsymbol{\sigma}}^{\text {mech }} \overline{\mathbf{F}}^{-T}
$$


In turn, the Maxwell stress $\overline{\boldsymbol{\sigma}}^{\text {maxw }}$ in the RVE is obtained via successive use of the chain rule and reads

$$
\begin{aligned}
\overline{\boldsymbol{\sigma}}^{\max w} & =\left(\frac{\partial \widetilde{w}}{\partial \overline{\mathbf{b}}} \cdot \frac{\partial \overline{\mathbf{b}}}{\partial \overline{\mathbf{F}}}\right) \overline{\mathbf{F}}^{T}= \\
& =\bar{\rho}_{0} \frac{\partial \widetilde{\phi}}{\partial \overline{\mathbf{b}}} \cdot \frac{\partial \overline{\mathbf{b}}}{\partial \overline{\mathbf{F}}}+\frac{\partial}{\partial \overline{\mathbf{b}}}\left[\frac{\bar{J}}{2 \mu_{0}} \overline{\mathbf{b}} \cdot \overline{\mathbf{b}}\right] \cdot \frac{\partial \overline{\mathbf{b}}}{\partial \overline{\mathbf{F}}}+\frac{1}{2 \mu_{0}}(\overline{\mathbf{b}} \cdot \overline{\mathbf{b}}) \frac{\partial \bar{J}}{\partial \overline{\mathbf{F}}}= \\
& =\frac{1}{\mu_{0}} \overline{\mathbf{b}} \otimes \overline{\mathbf{b}}-\frac{1}{2 \mu_{0}}|\overline{\mathbf{b}}|^{2} \mathbf{I}+(\overline{\mathbf{m}} \cdot \overline{\mathbf{b}}) \mathbf{I}-\overline{\mathbf{m}} \otimes \overline{\mathbf{b}}= \\
& =\overline{\mathbf{h}} \otimes \overline{\mathbf{b}}-\frac{\mu_{0}}{2}\left(|\overline{\mathbf{h}}|^{2}-|\overline{\mathbf{m}}|^{2}\right) \mathbf{I}, \quad \text { with } \quad \overline{\mathbf{S}}^{\max w}=\bar{J} \overline{\boldsymbol{\sigma}}^{\max w} \overline{\mathbf{F}}^{-T} .
\end{aligned}
$$

In the present case of an RVE, the above Maxwell stress serves to describe the magnetoelastic interactions between the magnetizable phases.

Next, using (3.9), (3.10) and (3.11) together with (3.13) and (3.18), one gets the intermediate result

$$
\bar{\rho}_{0} \frac{\partial \widetilde{\Phi}}{\partial \overline{\mathbf{F}}} \overline{\mathbf{F}}^{T}=\left.\bar{\rho}_{0} \frac{\partial \widetilde{\phi}}{\partial \overline{\mathbf{F}}}\right|_{\overline{\mathbf{b}}} \overline{\mathbf{F}}^{T}+\bar{J}(\overline{\mathbf{m}} \cdot \overline{\mathbf{b}}) \mathbf{I}-\bar{J} \overline{\mathbf{m}} \otimes \overline{\mathbf{b}}
$$

In view of equations (3.13), (3.14) and (3.17) and with reference to the sketch in Fig.3e, one readily shows that the Eulerian average mechanical traction is simply

$$
\overline{\mathbf{t}}=\left[\overline{\boldsymbol{\sigma}}-\overline{\boldsymbol{\sigma}}^{\max w}\right] \mathbf{n}=\left.\frac{\bar{\rho}_{0}}{\bar{J}} \frac{\partial \widetilde{\phi}}{\partial \overline{\mathbf{F}}}\right|_{\overline{\mathbf{b}}} \overline{\mathbf{F}}^{T} \mathbf{n} \equiv \overline{\boldsymbol{\sigma}}^{\text {mech }} \mathbf{n}, \quad \text { in } \quad \partial \mathcal{V}^{\#}
$$

or equivalently by additional use of (3.19), the average mechanical traction $\overline{\mathbf{T}}$ and average mechanical first Piola-Kirchhoff stress, $\overline{\mathbf{S}}^{\text {mech }}$, in the reference configuration become

$$
\overline{\mathbf{T}}=\left[\overline{\mathbf{S}}-\overline{\mathbf{S}}^{\text {maxw }}\right] \mathcal{N}=\left[\bar{\rho}_{0} \frac{\partial \widetilde{\Phi}}{\partial \overline{\mathbf{F}}}-\bar{J}(\overline{\mathbf{m}} \cdot \overline{\mathbf{b}}) \overline{\mathbf{F}}^{-T}+\bar{J}(\overline{\mathbf{m}} \otimes \overline{\mathbf{b}}) \overline{\mathbf{F}}^{-T}\right] \mathcal{N} \equiv \overline{\mathbf{S}}^{\text {mech }} \mathcal{N}, \quad \text { in } \quad \partial \mathcal{V}_{0}^{\#}
$$

The average mechanical traction $\overline{\mathbf{T}}$ defined in (3.21) is free from contributions from the Maxwell stresses present in the surrounding of the RVE (see Fig. 3b for a graphical illustration). The use of this definition is critical since it allows to analyze the magnetostriction resulting from the magnetoelastic interactions within the RVE without being affected by macroscopic geometry/shape effects (see Fig. 3).

Obviously, the operation in (3.21) is a non-trivial one since it would require solving the homogenization problem (3.5), computing all the necessary average fields, i.e., $\overline{\mathbf{F}}, \overline{\mathbf{S}}, \overline{\mathbf{b}}, \overline{\mathbf{h}}$ and $\overline{\mathbf{m}}$ and then carrying out the average traction condition in (3.21). To avoid such iterations, we propose in the following section an augmented variational principle that is capable of resolving "directly" the above-defined average traction condition and thus analyzing the homogenization problem in real time.

\section{Augmented variational principle}

In this section, we propose an augmented variational principle using the above described homogenized energy function and we show that this modification is necessary for applying the average mechanical traction in (3.21) by properly canceling out the average Maxwell stress on the periodic unit cell boundary, as discussed in the previous section in the context of Fig.3. This is done in order to be able to understand the underlying micro-mechanisms leading to the deformation of a composite magnetoelastic material lying inside a uniform average magnetic field and of course neglecting any magnetomechanical gradients arising due to specimen geometries present in a standard BVP. This can be achieved by considering the following conditions explained in detail in the following two subsections. 


\subsection{Treat the average Maxwell stress along interface of the RVE}

In order to impose continuity of the average Maxwell stress in the context of periodic homogenization, we subtract from the effective energy in (3.5) the term

$$
\widetilde{W}_{\operatorname{maxw}}(\overline{\mathbf{F}}, \overline{\mathbf{B}})=\frac{1}{2 \mu_{0} \bar{J}} \overline{\mathbf{F}} \overline{\mathbf{B}} \cdot \overline{\mathbf{F}} \overline{\mathbf{B}} .
$$

The term $\widetilde{W}_{\text {maxw }}$ in (4.1) corresponds to the effective magnetic energy due to the average magnetic field and average deformation gradient in the RVE and by subtracting it from the effective energy, one effectively enforces continuity of the first two terms of the average Maxwell stress in equation (3.17). This term alone gives rise to deformation fields $\overline{\mathbf{F}}$ under application of a magnetic field $\overline{\mathbf{B}}$. If one retains the above term in the effective energy, this leads to an unrealistic fictitious deformation of the solid - even when the solid is not magnetic - as if the magnetic poles were attached on the boundary of the solid exerting an additional stressing to the body due to their mutual attraction.

With regard to this observation, in the context of electroactive solids, the electrodes are attached to the body and deform with its boundary while the attractive forces between the electrodes contribute to the deformation of the body itself, as already discussed in Fig. 1b. It is therefore obvious that one could keep the term (4.1) in the estimation of the energy $\widetilde{W}$ if the interest is on electroactive response (see for instance Lefèvre and Lopez-Pamies (2016a,b)). But again in that case, one measures both the electromechanical coupling of the material as well as the contribution of the electrodes upon the overall deformation of the composite.

It should be stressed here that the "mathematical" homogenization problem itself in (3.5) is well-posed and one could homogenize and subtract the $\widetilde{W}_{\text {maxw }}$ energy afterwards, i.e., the effective energy function is independent of the boundary conditions. Nonetheless, this would not allow the direct numerical simulation and observation of the micro-deformation mechanisms in the given microstructures.

\subsection{Application of the Eulerian magnetic field in the unit cell}

The experimental configuration in Fig. 1a implies the application of a Eulerian magnetic field. As a consequence, at the scale of the RVE one should apply/control the average Eulerian magnetic field $\overline{\mathbf{b}}$ instead of the Lagrangian one, $\overline{\mathbf{B}}$. In other words, if one lies at a given point inside the magnetoelastic solid, the average (background) field at the RVE scale shall not follow the deformation of the magnetizable body and thus constitutes (by nature) a Eulerian measure. The presence of a "periodic" magnetizable body, in turn, inside this field induces a perturbation field $\widetilde{\mathbf{B}}$ (or equivalently $\widetilde{\mathbf{b}}$ ) which will be periodic with zero average. This has significant implications in the resulting deformation of the periodic medium as well as the relevant periodic boundary conditions since, in this case, one has to apply/control

$$
\bar{J}^{-1} \overline{\mathbf{F}} \overline{\mathbf{B}}=\overline{\mathbf{b}} \equiv \mathbf{b}_{a}
$$

and not $\overline{\mathbf{B}}$. Here, $\mathbf{b}_{a}$ is a uniform vector field with time-evolving amplitude and is used to apply the average Eulerian magnetic field $\overline{\mathbf{b}}$. This last expression constitutes a nonlinear constraint on the average variables $\overline{\mathbf{F}}$ and $\overline{\mathbf{B}}$ and consequently leads to a non-proportional loading in the space $\overline{\mathbf{F}}-\overline{\mathbf{B}}$. While such a condition seems non-standard in homogenization theories, it is merely a specific type of loading and does not alter anything in the original homogenization problem (3.5). Nonetheless, it is the one that will lead to a correct interpretation of the simulated microstructures and the corresponding micro-deformation mechanisms resulting by the short and long range interaction of magnetic phases in the presence of an average Eulerian magnetic field $\overline{\mathbf{b}}$.

Again, if one is only interested in the analytical estimation of the effective energy in equation (3.5) then neither of the above conditions need to be taken into account a priori (Ponte Castañeda and Galipeau, 2011; Lopez-Pamies, 2014). The focus, however, in this study is to extract the magnetoelastic response of a material without the effect of the macroscopic specimen shape, i.e., the magnetization and magnetostriction response due to the microstructural interactions at the RVE scale. If the above terms are not taken into account during the numerical simulation, one could estimate $\widetilde{W}$ in (3.5) by scanning the entire $\overline{\mathbf{F}}-\overline{\mathbf{B}}$ space and then 
choose only the points that satisfy (4.2) as well as the traction (3.20). Nonetheless, in that case, one would not have access to the underlying micro-deformation mechanisms that lead to the overall magnetostriction of the composite, whereby a large amount of simulations would be needed to cover all possible loading states. The risk of misinterpretation of the resulting magnetostriction is also present in this last approach. In this regard, making contact with the corresponding electromechanical setup in Fig. 1b, one easily observes that due to the fact that the electrodes and hence the applied electric field follow the deformation of the solid, the natural applied field is now the Lagrangian counterpart of $\overline{\mathbf{B}}$ in electrostatics (i.e., the electric displacement $\overline{\mathbf{D}})$ and not the Eulerian one, $\overline{\mathbf{b}}$. In this case, however, the obtained electrostriction is a combined result of the microstructural interactions as well as the attractive forces induced by the electrodes.

\subsection{The final expression for the augmented variational principle}

We show next that use of the above-described conditions lead readily to imposing the average traction condition (3.20) or (3.21). Thus, by subtracting the energy (4.1) and imposing the constraint (4.2) in the original effective energy formulation (3.5), one readily obtains an augmented variational formulation for a homogenized medium which reads

$$
\mathcal{P}(\overline{\mathbf{F}}, \overline{\mathbf{B}})=\widetilde{W}(\overline{\mathbf{F}}, \overline{\mathbf{B}})-\frac{1}{2 \mu_{0} \bar{J}} \overline{\mathbf{F}} \overline{\mathbf{B}} \cdot \overline{\mathbf{F}} \overline{\mathbf{B}}+\frac{\bar{J}}{2 \mu_{0} \xi}\left|\bar{J}^{-1} \overline{\mathbf{F}} \overline{\mathbf{B}}-\mathbf{b}_{a}\right|^{2}
$$

Here, $\widetilde{W}$ is given by (3.5), while, $\xi$ is a penalty factor serving to enforce the constraint (4.2). In practice, it suffices to set it equal to a very small number (e.g., $\xi=10^{-7}$ ). The addition of $\mu_{0}$ in the last term of equation (4.3) allows for correct dimensionalization of this term letting $\xi$ serve as a simple constant without units. The above-described formulation can be seen as an alternative numerically efficient way to estimate the free energy $\widetilde{\Phi}$, defined in equation (3.9), together with a properly defined average mechanical traction condition and a prescribed Eulerian magnetic field $\overline{\mathbf{b}}$.

Following Michel et al. (1999), one could apply prescribed mechanical prestresses $\overline{\mathbf{S}}_{0}^{\text {mech }}$ (or alternatively $\left.\overline{\boldsymbol{\sigma}}_{0}^{\text {mech }}=\bar{J}^{-1} \overline{\mathbf{S}}_{0}^{\text {mech }} \overline{\mathbf{F}}^{T}\right)$ via an additional term $\overline{\mathbf{S}}_{0}^{\text {mech }} \cdot(\overline{\mathbf{F}}-\mathbf{I})$, thus modifying accordingly the variational principle (4.3) as

$$
\mathcal{P}_{S}(\overline{\mathbf{F}}, \overline{\mathbf{B}})=\widetilde{W}(\overline{\mathbf{F}}, \overline{\mathbf{B}})-\frac{1}{2 \mu_{0} \bar{J}} \overline{\mathbf{F}} \overline{\mathbf{B}} \cdot \overline{\mathbf{F}} \overline{\mathbf{B}}+\frac{\bar{J}}{2 \mu_{0} \xi}\left|\bar{J}^{-1} \overline{\mathbf{F}} \overline{\mathbf{B}}-\mathbf{b}_{a}\right|^{2}-\overline{\mathbf{S}}_{0}^{m e c h} \cdot(\overline{\mathbf{F}}-\mathbf{I}) .
$$

The term, $\overline{\mathbf{S}}_{0}^{\text {mech }}$ is a given quantity related directly to the average traction $\overline{\mathbf{T}}$, defined in equation (3.21). This last term is included only if one is interested in overall mechanical prestresses.

Noting next that $\overline{\mathbf{F}}$ and $\overline{\mathbf{B}}$ are independent variables in the variational principle (4.3), one can show that variation of $\mathcal{P}$ (it is the same for $\mathcal{P}_{S}$ ) with respect to those quantities satisfies the average conditions (3.8), (3.11) and (3.21), identically. To show this, we consider, first, that

$$
\delta \mathcal{P}=\frac{\partial \mathcal{P}}{\partial \overline{\mathbf{F}}} \cdot \delta \overline{\mathbf{F}}+\frac{\partial \mathcal{P}}{\partial \overline{\mathbf{B}}} \cdot \delta \overline{\mathbf{B}}=0, \quad \text { in } \quad \mathcal{V}_{0}^{\#}
$$

Since, $\delta \overline{\mathbf{F}}$ and $\delta \overline{\mathbf{B}}$ are independent variations, one has that each prefactor multiplying these variations should be identically zero. In this regard, one can easily show that

$$
\frac{\partial \mathcal{P}}{\partial \overline{\mathbf{B}}}=\frac{\partial \mathcal{P}_{S}}{\partial \overline{\mathbf{B}}}=\frac{\partial \widetilde{W}}{\partial \overline{\mathbf{B}}}-\frac{1}{\mu_{0} \bar{J}} \overline{\mathbf{F}}^{T}(\overline{\mathbf{F}} \overline{\mathbf{B}})+\frac{1}{\mu_{0} \xi \bar{J}}\left(\overline{\mathbf{F}} \overline{\mathbf{B}}-\mathbf{b}_{a}\right) \overline{\mathbf{F}}=\left(\overline{\mathbf{h}}-\frac{1}{\mu_{0}} \overline{\mathbf{b}}+\frac{\bar{J}}{\mu_{0} \xi \bar{J}}\left(\overline{\mathbf{b}}-\mathbf{b}_{a}\right)\right) \overline{\mathbf{F}}=\mathbf{0},
$$

in $\mathcal{V}_{0}^{\#}$. This implies that the penalty term in (4.3) gives rise to terms that involve the average magnetization. This is readily seen by observation of the last equation (4.6) and direct comparison with relation (3.11), such that one gets

$$
\overline{\mathbf{m}}=\frac{1}{\mu_{0} \xi}\left(\overline{\mathbf{b}}-\mathbf{b}_{a}\right) .
$$


It is noted here that the above expression for $\overline{\mathbf{m}}$ is exactly equivalent to the original definition in (3.11)), which provides a physical interpretation to the penalty term of equation (4.4). In addition, one could further point out that if in equation (4.3) one uses a Lagrange multiplier, i.e., replacing the penalty term $\bar{J}\left|\bar{J}^{-1} \overline{\mathbf{F}} \overline{\mathbf{B}}-\mathbf{b}_{a}\right|^{2} /\left(2 \mu_{0} \xi\right)$ with $\bar{J} \overline{\boldsymbol{\lambda}} \cdot\left(\bar{J}^{-1} \overline{\mathbf{F}} \overline{\mathbf{B}}-\mathbf{b}_{a}\right) / \mu_{0}$, then it can be readily shown that the Lagrange multiplier $\bar{\lambda}$ is directly identified with the average magnetization, i.e., $\overline{\boldsymbol{\lambda}}=\overline{\mathbf{m}}$. In this work, we use a penalty method in order to preserve the minimum character of the variational principle (4.3) for use in stability analysis in future studies.

Next, by considering the variation with respect to $\overline{\mathbf{F}}$ in (4.3) and use of definition (4.7), one gets (after some tedious but otherwise straightforward algebra)

$$
\begin{aligned}
\frac{\partial \mathcal{P}_{S}}{\partial \overline{\mathbf{F}}}=\frac{\partial \mathcal{P}}{\partial \overline{\mathbf{F}}}-\overline{\mathbf{S}}_{0}^{\text {mech }} & =\bar{J}\left(\overline{\boldsymbol{\sigma}}-\frac{1}{\mu_{0}} \overline{\mathbf{b}} \otimes \overline{\mathbf{b}}+\frac{1}{2 \mu_{0}}|\overline{\mathbf{b}}|^{2} \mathbf{I}-(\overline{\mathbf{m}} \cdot \overline{\mathbf{b}}) \mathbf{I}+\overline{\mathbf{m}} \otimes \overline{\mathbf{b}}-\overline{\boldsymbol{\sigma}}^{\text {mech }}\right) \overline{\mathbf{F}}^{-T}= \\
& =\bar{J}\left(\overline{\boldsymbol{\sigma}}-\overline{\boldsymbol{\sigma}}^{\text {maxw }}-\overline{\boldsymbol{\sigma}}_{0}^{m e c h}\right) \overline{\mathbf{F}}^{-T}=\bar{J}\left(\overline{\boldsymbol{\sigma}}^{\text {mech }}-\overline{\boldsymbol{\sigma}}_{0}^{\text {mech }}\right) \overline{\mathbf{F}}^{-T}= \\
& =\overline{\mathbf{S}}-\overline{\mathbf{S}}^{\text {maxw }}-\overline{\mathbf{S}}_{0}^{\text {mech }}=\overline{\mathbf{S}}^{\text {mech }}-\overline{\mathbf{S}}_{0}^{\text {mech }}=\mathbf{0}, \quad \text { in } \mathcal{V}_{0}^{\#} .
\end{aligned}
$$

Here, $\overline{\boldsymbol{\sigma}}=\overline{\boldsymbol{\sigma}}^{\text {mech }}+\overline{\boldsymbol{\sigma}}^{\text {maxw }}$ and $\overline{\boldsymbol{\sigma}}^{\text {maxw }}$ have been defined in equations (3.13)-(3.14) and (3.17), respectively. This last relation proves that the proposed variational principle (4.3) is equivalent to applying directly the average mechanical traction, $\overline{\mathbf{t}}$ or $\overline{\mathbf{T}}$ (c.f. (3.20) and (3.21), respectively), or equivalently the average stress $\overline{\boldsymbol{\sigma}}^{\text {mech }}$ or $\overline{\mathbf{S}}^{\text {mech }}$ (c.f. (3.14) and (3.20), respectively).

Finally, it should be noted that the above proposed augmented variational principle in equation (4.3) is also valid in the more general context of boundary value problems if one notices that it may describe the response of any material point at the macroscale (see for instance green box in Fig. 3a). In that case, the average $\overline{\mathbf{b}}$ field is the true magnetic field in the macroscale as discussed in the context of Fig. 3a. This observation will become more clear in Appendix A, where we show that the variational principle (4.3) is equivalent to that of Kankanala and Triantafyllidis (2004), James and Kinderlehrer (1993) and Liu et al. (2006) under certain properties of the energy functions $\phi^{(r)}(\mathbf{F}, \mathbf{b})$.

\section{Results : Classical microstructures}

In this section, we discuss the effective magnetomechanical response of two-phase systems obtained for a wide range of microstructures subjected to a simple set of loading conditions, as will be described in the following sections. We also focus on simple energy functions for the constituents. We consider that both matrix and particles can be described by the simple energy functions discussed in Section 2.1. Specifically, we consider that, at the constituents level, the total magnetomechanical energy can be decoupled into a purely mechanical and a purely magnetic part (c.f. (2.9) and (2.11)), i.e.,

$$
\begin{aligned}
\rho_{0}^{(r)} \Phi^{(r)}(\mathbf{F}, \mathbf{B})= & \frac{G}{2}(\mathbf{F} \cdot \mathbf{F}-3-2 \ln J)+\frac{G^{\prime}}{2}(J-1)^{2}+ \\
& \frac{J \mu_{0}\left(m_{s}^{(r)}\right)^{2}}{3 \chi^{(r)}}\left\{\ln \left[\frac{3 \chi^{(r)}}{\mu_{0} m_{s}^{(r)} J} \sqrt{\mathbf{F B} \cdot \mathbf{F B}}\right]-\ln \left[\sinh \left(\frac{3 \chi^{(r)}}{\mu_{0} m_{s}^{(r)} J} \sqrt{\mathbf{F B} \cdot \mathbf{F B}}\right)\right]\right\},
\end{aligned}
$$

where the the superscript $r=1,2, \ldots$ serves to label each phase. The above choice of the energy function is a valid assumption provided that neither of the phases exhibits a significant magnetomechanical coupling. That is usually true for the matrix which is a non-magnetic polymeric material or the air/void. The particle on the other hand, which is usually made of iron can exhibit intrinsic magnetostriction but an extremely small one (e.g., strains in the order of $10^{-4}-10^{-6}$ or $p p m$ ). This intrinsic particle magnetostriction is significantly smaller than the strains obtained for the MRE composite (e.g., strains in the order of $10^{-2}-10^{0}$ ). Thus, the simple decoupled energies described in (5.1) may also be used for the particle. It is noted of course that the variational principle, defined in (4.3), can deal with any coupled energy function if required by the application at hand. 
In the following, we mostly make use of the same set of material parameters unless stated otherwise. We choose to work with a matrix that resembles a soft silicone material and thus exhibits a shear modulus in the order of $10-1000 \mathrm{KPa}$ and zero magnetic susceptibility. In turn the particle is treated as rigid, since it usually has a shear modulus of $100-200 \mathrm{GPa}$, and magnetic susceptibility $\chi \rightarrow 1$ or $\chi_{v} \rightarrow \infty$. For numerical convergence and clarity of the results, we have chosen the following set of material parameters in Table 1:

\begin{tabular}{|c|c|c|c|c|c|c|c|c|}
\hline & $G(\mathrm{MPa})$ & $G^{\prime} / G$ & $E(\mathrm{MPa})$ & $\nu$ & $\chi$ & $\chi_{v}$ & $\mu_{0} m_{s}(\mathrm{~T})$ & $\mu_{0}\left(\mu \mathrm{N} \cdot \mathrm{A}^{-2}\right)$ \\
\hline Matrix & 0.05 & 100 & 0.1495 & 0.495 & 0.0 & 0.0 & - & $4 \pi 10^{-1}$ \\
\hline Particle & 50 & 100 & 149.5 & 0.495 & 0.995 & 200 & 2 & $4 \pi 10^{-1}$ \\
\hline
\end{tabular}

Table 1: Material parameters corresponding to a silicon matrix and carbonyl iron particles.

Note that the shear modulus of the particle is chosen as 1000 times larger than that of the matrix. This contrast is sufficiently large to promote a rigid response of the particle and at the same time allow for very good numerical convergence. The volumetric term $(J-1)^{2}$ in $(2.9)$ is under-integrated using a single quadrature point to avoid volumetric locking effects. The rest of the terms are fully integrated by using a $2 \times 2$ quadrature rule. Furthermore, the units of the shear modulus is in MPa and hence the magnetic permeability in vacuum is written in units of micro-Newton over Amperes square. The calculations are carried out by building a user-element subroutine in the finite element package FEAP. The present code has been checked independently with other commercial codes for the purely mechanical part, with the analytical solution of a circle in an infinite domain subjected to a purely magnetic load (i.e., solution of the Laplace equation), a one element calculation and analytical results, and with the analytical exact results of Tian et al. (2012) and Lefèvre and Lopez-Pamies (2015) for the coupled response at relatively small deformations ${ }^{2}$. In all cases, the obtained results were found to be in excellent agreement.

For the rest of the study, we set the normal components of the mechanical traction to be zero on the boundary of the unit cell, while the shear components of the deformation gradient are blocked. The Eulerian magnetic field is always applied in the 2 -direction. Those conditions read

$$
\bar{S}_{11}^{\text {mech }}=\bar{S}_{22}^{\text {mech }}=0, \quad \bar{F}_{12}=\bar{F}_{21}=0, \quad \overline{\mathbf{b}}=\mathbf{b}_{a}=\bar{b} \mathbf{e}^{(2)}, \quad \text { in } \quad \mathcal{V}_{0}^{\#} .
$$

For more details on the application of the boundary conditions, the reader is referred to Appendix B. It is recalled that the applied Eulerian magnetic field $\bar{b}$ is applied directly via the third penalty term in equation (4.3). No prestresses are considered in this study.

\subsection{A single circular particle in a square unit cell}

We first analyze the single-particle square unit cell shown in the inset of Fig. 4a. As already discussed in the literature, intuitively we expect particles to be attracted in the direction of the applied magnetic field (i.e., in $X_{2}$ in the present example) and thus leading to an overall contraction (negative magnetostriction). Figure 4 shows the average magnetization $\bar{m}_{2} / m_{s}$ (where $m_{s}$ is the saturation magnetization of the particle) and the average magnetostriction $\bar{\lambda}_{2}-1$ as a function of the applied normalized average Eulerian magnetic field $\bar{b} / \mu_{0} m_{s}$ for various particle volume fractions $c=5,10,15,25,35,40 \mathrm{vol} \%$. As expected in Fig. 4a, the average magnetization response exhibits a monotonic increase with the volume fraction. In turn, the corresponding average magnetostriction, shown in Fig. 4b, is less intuitive. While the initial curvature (see inset in this figure) increases with the volume fraction, at higher magnetic fields we observe a crossover of the curves for $c>25 \mathrm{vol} \%$. This crossover at finite magnetic fields implies that as we further increase the volume fraction, and thus increasing the magnetic interactions between neighboring particles in neighboring unit cells, the corresponding overall magnetomechanical coupling weakens. This can be explained by the observation that by increasing the volume fraction, one increases effectively the pure mechanical stiffness of the composite thus hindering further increase of magnetostriction beyond a certain volume fraction. As we

\footnotetext{
${ }^{2}$ The referenced work is in the context of electroactive polymers, however, it is by now well known that the electroactive and magnetoactive problems resemble apart from the applied boundary conditions.
} 
a)



b)

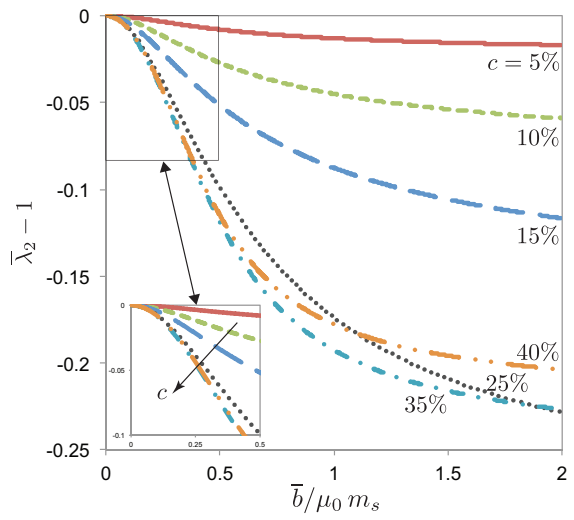

Figure 4: Macroscopic response of a square periodic unit cell comprising one circular rigid magnetizable inclusion set at the center of the square. The composite is subjected to a magnetic field in the $X_{2}$ direction and zero overall mechanical traction. (a) Average magnetization and (b) average magnetostriction as a function of the normalized average Eulerian magnetic field $\bar{b} / \mu_{0} m_{s}$ for various particle volume fractions $c=5,10,15,25,35,40 \mathrm{vol} \%$.

will observe further below this transition point depends on the choice of the unit cell and on other material parameters and one cannot make more general statements at this point, except that increase of volume fraction does not necessarily lead to increase of the magnetostriction, even if we obtain an increase in the effective magnetic susceptibility.

\subsection{Single elliptical particle}

In the following, we consider an elliptical particle with aspect ratio $w=1 / 4$ embedded in a square unit cell, as shown in the inset of Fig. 5. The volume fraction of the particle is $c=15 \mathrm{vol} \%$. The longest axis of the ellipsoid forms angle $\theta_{0}$ with respect to the $X_{1}$ laboratory axis and the applied magnetic field $\overline{\mathbf{b}}$ is always in the $X_{2}$ direction. We, then, study the effect of the initial particle orientation angle $\theta_{0}$ with respect to the applied magnetic field $\overline{\mathbf{b}}$. In Fig. $5 \mathrm{a}$, we observe a monotonic increase of the magnetization $\bar{m}_{2} / m_{s}$ as $\theta_{0}$ goes from $0^{\circ}$ to $90^{\circ}$ in the direction of the applied magnetic field $X_{2}$. In turn, the magnetization in the transverse direction, i.e., $\bar{m}_{1} / m_{s}$, is non-monotonic and negative, as shown in the inset of Fig. 5a. Initially, $\bar{m}_{1} / m_{s}$ grows in amplitude while it exhibits a maximum which is a function of the orientation angle $\theta_{0}$. As the ellipse rotates more to align with the applied magnetic field the $\bar{m}_{1}$ component decreases again in amplitude to approach zero. On the other hand, the $\bar{m}_{2} / m_{s}$ magnetization response at large magnetic fields saturates at the same asymptote irrespective of the initial angle $\theta_{0}$.

Next, it is worth discussing the particular case of $\theta=0^{\circ}$. In this case, in Fig. 5, we use a black dot to denote the loss of positive definiteness of the global stiffness matrix in the FE calculation ${ }^{3}$. This indicates the presence of a negative eigenvalue and possible bifurcation at this point. In this case of $\theta_{0}=0^{\circ}$, the MRE contracts in the direction $X_{2}$ of the applied field $\overline{\mathbf{b}}$ as intuitively expected. Beyond the black dot the FE result remains along this principal solution, which is not any more minimizing the total energy of the system and thus is not correct, but is shown here for completeness. In order to investigate further this result, we restrict attention to the same single unit cell and consider a small deviation from the horizontal configuration by setting $\theta_{0}=5^{\circ}$ (as shown in the cntours of Fig. 6). By looking at the $\bar{\lambda}_{2}-1$ curves in Fig. 5b, we observe a contraction along the $X_{2}$ direction, with the $\theta_{0}=5^{\circ}$ curve initially coinciding with

\footnotetext{
${ }^{3}$ This is straightforward to conclude from our calculations since we use a minimum variational principle, i.e., by use of a vector potential formulation and penalty terms. That result is less trivial to attain if use is made of the well-known scalar potential formulation or a mixed pressure FE formulation, since they both lead to a min-max (saddle point) variational principle (Miehe et al., 2016).
} 
a)

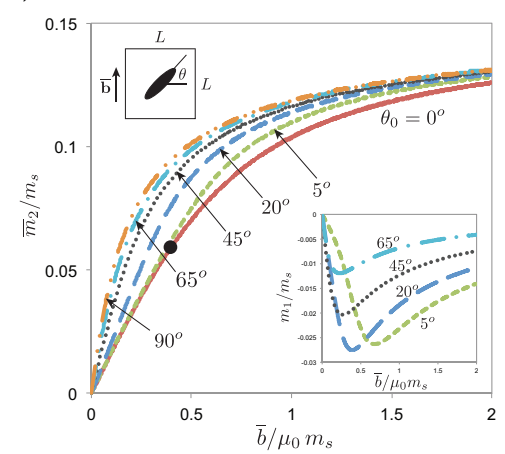

b)

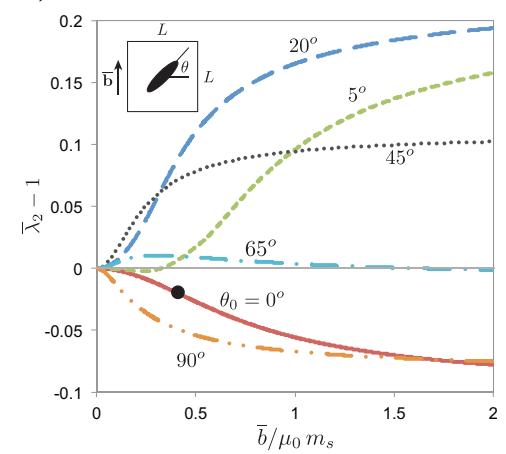

c)

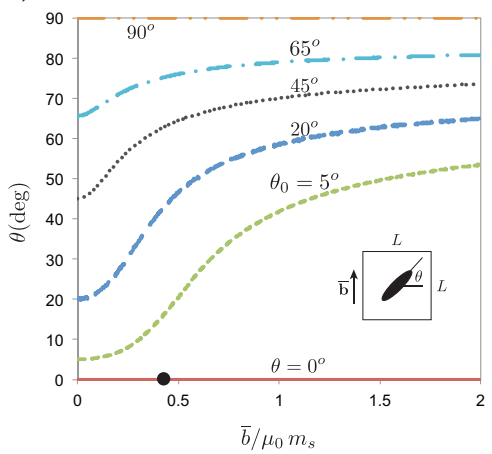

Figure 5: Macroscopic response of a square periodic unit cell with external aspect ratio comprising elliptical rigid magnetizable inclusions whose longest axis forms an angle $\theta$ woth respect to the $X_{1}$ direction. The composite is subjected to a magnetic field in the $X_{2}$ direction and zero overall mechanical traction. (a) Average magnetization, (b) average magnetostriction and (c) orientation angle $\theta$ of the elliptical particle as a function of the normalized average Eulerian magnetic field $\bar{b} / \mu_{0} m_{s}$ for various orientation angles $\theta=0,5,20,45,65,90^{\circ}$. The volume fraction of the inclusion is $c=15 \mathrm{vol} \%$. The inset in part (a) shows the magnetization $\bar{m}_{1} / m_{s}$ transversely to the applied magnetic field and appears to be non-monotonic.

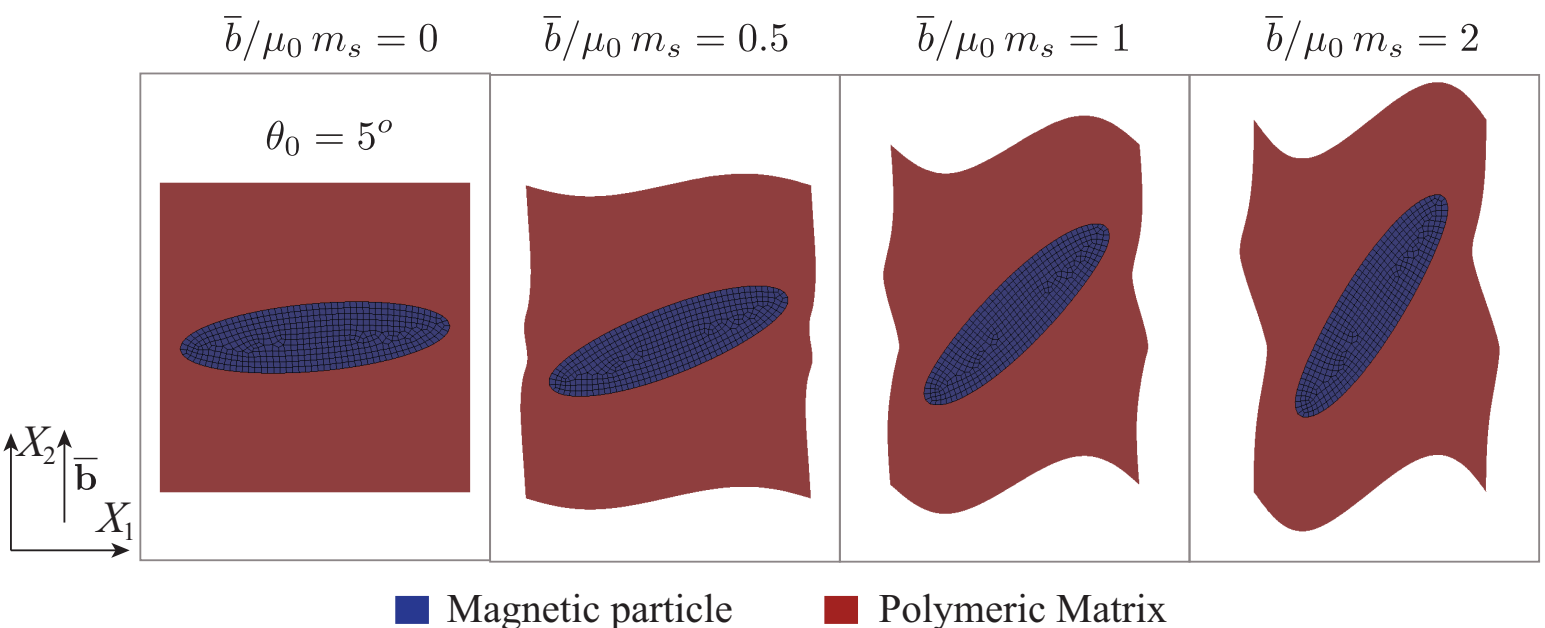

Figure 6: Deformed unit cell comprising a single elliptical particle with aspect ratio $w=1 / 4$ and volume ftaction $c=15 \mathrm{vol} \%$. The initial angle of the particle is set to $\theta=5^{\circ}$. The applied magnetic field $\bar{b} / \mu_{0} m_{s}$ is applied in $X_{2}$ direction. This induces a significant rotation of the particle, which is unfavorably positioned with respect to the magnetic field and thus tends to align it. The induced rotation leads to significant elongation along the direction of the magnetic field.

the one for $\theta_{0}=0^{\circ}$. Nevertheless, rather rapidly, the $\theta_{0}=5^{\circ}$ deviates from the one for $0^{\circ}$ resulting in a significant extension of the unit cell, i.e., $\bar{\lambda}_{2}-1>0$. This extension is a direct consequence of the rotation of the longest axis of the ellipse in an effort to align with the applied magnetic field in the $X_{2}$ direction as shown in Fig. 5c (see also the studies of Galipeau and Ponte Castañeda (2013) and Galipeau et al. (2014) in these cases, as well as that of Lefèvre and Lopez-Pamies (2015) in the electroactive context). By further increase of the initial angle $\theta_{0}=20^{\circ}$, we obtain an extension of the MRE along the applied magnetic field $\overline{\mathbf{b}}$. The magnetostriction response, however, is not monotonic as a function of $\theta_{0}$, as clearly shown in Fig. 5b. This is exhibited by increasing further the particle orientation angle to $\theta_{0}=45^{\circ}$. In that case, the initial curvature is higher than those for $\theta_{0}=5^{\circ}$ and $20^{\circ}$ but the overall positive magnetostriction saturates very 
fast leading to a lower saturation strain than that obtained for $\theta_{0}=5^{\circ}$ and $20^{\circ}$ at moderate and large magnetic fields. In turn, further increase of the angle $\theta_{0}$ to $65^{\circ}$ and $90^{\circ}$ leads again to pure contraction along the direction of $\overline{\mathbf{b}}$, i.e., $X_{2}$ and an overall negative magnetostriction.

The evolution of the orientation angle of the particle longest axis as a function of the applied field $\bar{b} / \mu_{0} m_{s}$ is shown in Fig. $5 \mathrm{c}$. The particle with an initial angle $\theta_{0}=5^{\circ}$ shows a fastest rate of rotation at small magnetic fields and reaches a slow saturation at higher ones. The rate of rotation decreases with increase of the initial angle $\theta_{0}$. The rotation of the ellipse exhibits a monotonic dependence on the the initial angle $\theta_{0}$, similar to the magnetization response. For completeness and illustration purposes, in Fig 6 , we show snapshots of the deformed unit cell corresponding to an initial angle $\theta_{0}=5^{\circ}$. In these contours, one can easily observe the large average straining of the unit cell induced by the rotation of the ellipse. In passing, it is rather intuitive from those contours that if one allows for non-zero shear deformations, i.e., $\bar{F}_{12} \neq 0$ and/or $\bar{F}_{21} \neq 0$, then average shear strains are induced due to the rotation of the particle and that could also be seen as an interesting deformation mechanism for magnetoelastic devices. Such a work is underway.

\subsection{Unstructured multi-particle isotropic microstructures}

In this section, we analyze the local as well as the macroscopic response of unstructured isotropic MREs comprising a large number of monodisperse (equi-sized) particles randomly and uniformly distributed in the unit cell (see Fig. 7). We first focus on a single volume fraction $c=25 \mathrm{vol} \%$ and carry out a convergence analysis on the number of particles needed in the unit cell to obtain a representative volume element, i.e., to obtain average quantities that tend to be independent of the realization. We again consider the boundary conditions described in equation (5.2).

The particles are initially distributed following a random adsorption algorithm (RSA) (Torquato, 2002). Following earlier work (see for instance Segurado and Llorca (2002), López Jiménez (2014) and Lopez-Pamies et al. (2013)), the positions of the particles centers are obtained randomly, and are rejected if the distance to any of the already allocated particles is less than a given limit. In this work, the minimum distance adopted is 1.05 times the diameter. Particles that lie at the edge of the unit cell are periodically reproduced in the unit cell in order to obtain a periodic microstructure. Finally, a particle is also rejected if the distance between its center and the edge of the RVE is in the range of $0.95-1.05 \%$ of the diameter. These conditions are sufficient to provide isotropic responses as well as easy-to-mesh realizations.


Figure 7: Undeformed unit cells with unstructured isotropic distributions of particles and volume fraction $c=25 \mathrm{vol} \%$. We show four unit cells with number of particles $N_{p}=120,200,300,500$.

Figure 8 shows the effect of the number of particles upon the response of isotropic unit cells for a given volume fraction $c=25 \mathrm{vol} \%$. We consider five different numbers of particles $N_{p}=60,120,200,300,400,500$ (except in part (c) of this figure where additional numbers of particles are used) and five different realizations for each one of them (i.e., a total of 25 calculations). As easily observed in Fig. 8a, the magnetization response is already converged for $N_{p}=60^{4}$. Yet, the corresponding magnetostriction response, which is found to be

\footnotetext{
${ }^{4}$ The corresponding mechanical response (without magnetic fields) under uniaxial tension loadings is also converged at $N_{p}=60$ as shown by López Jiménez (2014) but is not shown here since it is a rather known result
} 
a)



b)

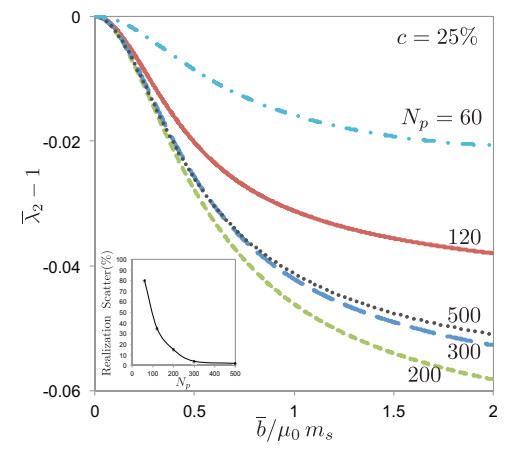

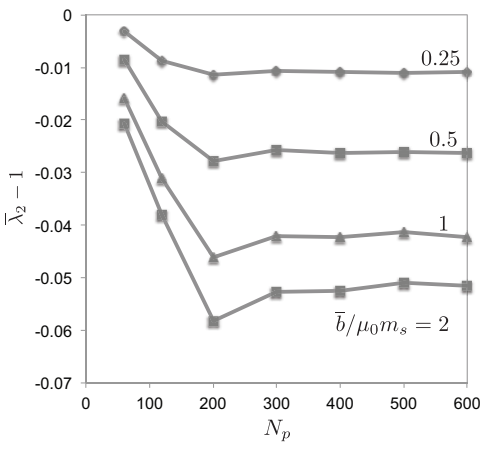

Figure 8: Macroscopic response of unstructured multi-particle isotropic periodic square unit cells comprising circular rigid magnetizable inclusions randomly distributed in the unit cell at a volume fraction of $c=25 \mathrm{vol} \%$. The composite is subjected to a magnetic field in the $X_{2}$ direction and zero average normal mechanical tractions. (a) Average magnetization and (b) average magnetostriction as a function of the normalized average Eulerian magnetic field $\bar{b} / \mu_{0} m_{s}$ for various number of particles $N_{p}$.

compressive in Fig. 8b,c, is highly sensitive to $N_{p}$ in the present case. The inset graph in Fig. 8b indicates the relative scatter induced by the different realizations used to obtain the response as a function of $N_{p}$ while Fig. $8 \mathrm{c}$ shows the $\bar{\lambda}_{2}-1$ as a function of $N_{p}$ for different levels of the applied magnetic field $\bar{b} / \mu_{0} m_{s}$. For $N_{p}=60, \bar{\lambda}_{2}-1$ gets the lowest values while the corresponding scatter is extremely large reaching values of $80 \%$ (see inset in part (b)). In fact, in this case of low number of particles some realizations give extension of the MRE (i.e., positive magnetostriction) and some give contraction (i.e., negative magnetostriction), thus inducing a significant scatter of the response and a non-converged volume element. Convergence of the magnetostriction response is achieved as we increase $N_{p} \geq 300$ particles, as easily seen in Fig. 8c. The need for high number of particles appears to be partially related to the fact that the magnetostriction response is ab initio a nonlinear function (initially quadratic) of the applied magnetic field. This non-linearity, albeit a weak one, requires a large number of particles in the unit cell in order to reach representativity. Other possible reasons for this high number of particles needed for convergence are also the prescribed boundary conditions, i.e., the application of the Eulerian magnetic field and not the Lagrangian one as well as the rather low shear modulus of the matrix phase used this example (see Table 1).

Figure 9 shows contours in the deformed configuration of the local normalized magnetic field $b_{2} / \mu_{0} m_{s}$ (top row) and of the local Green-Lagrange strain $E_{22}$ (bottom row) in the direction of the applied magnetic field at the instant $\bar{b} / \mu_{0} m_{s}=2$. The contours corresponding to $b_{2} / \mu_{0} m_{s}$ indicate the formation of particle chains along the applied magnetic field, while they reveal the strong interaction of neighboring particles in the same direction.

A very interesting observation can be made by focusing on the bottom row of Fig. 9, where we show the Green-Lagrange strain $E_{22}$, i.e., the component in the direction $X_{2}$ of the applied magnetic field. While the overall average magnetostriction in that case is negative (i.e., $\bar{E}_{22} \sim-0.05<0$ ), i.e., contraction of the unit cell along $X_{2}$ (as already shown in Fig. 8b), we observe regions of very high tensile strain $E_{22}>0.6$ (in red color). In these regions particles tend to move in directions perpendicular to the applied magnetic field in order to form particle chains similar to the observations made in Danas et al. (2012). Such high tensile strains, in turn, are expected to lead to debonding of the particles from the matrix and thus lead to an overall reduction of the magnetomechanical coupling and degradation of the MRE itself. These high local strains are again the reason for severe mesh distortion locally and subsequent cease of the calculations. To achieve highest strains, one could resort to polygonal elements (Talischi et al., 2010; Chi et al., 2016) or mesh-free techniques (Nayroles et al., 1992; Belytschko et al., 1994) but this goes beyond the scope of the present paper.

We conclude this section by showing in Fig. 10 the effect of particle volume fraction upon the effective 


$$
N_{p}=120 \quad N_{p}=200 \quad N_{p}=300 \quad N_{p}=500
$$

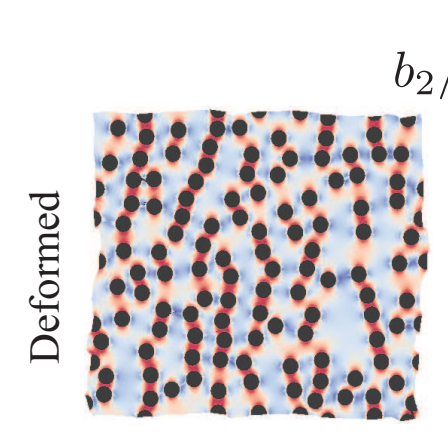

1.5

2.0 2.5 3.0
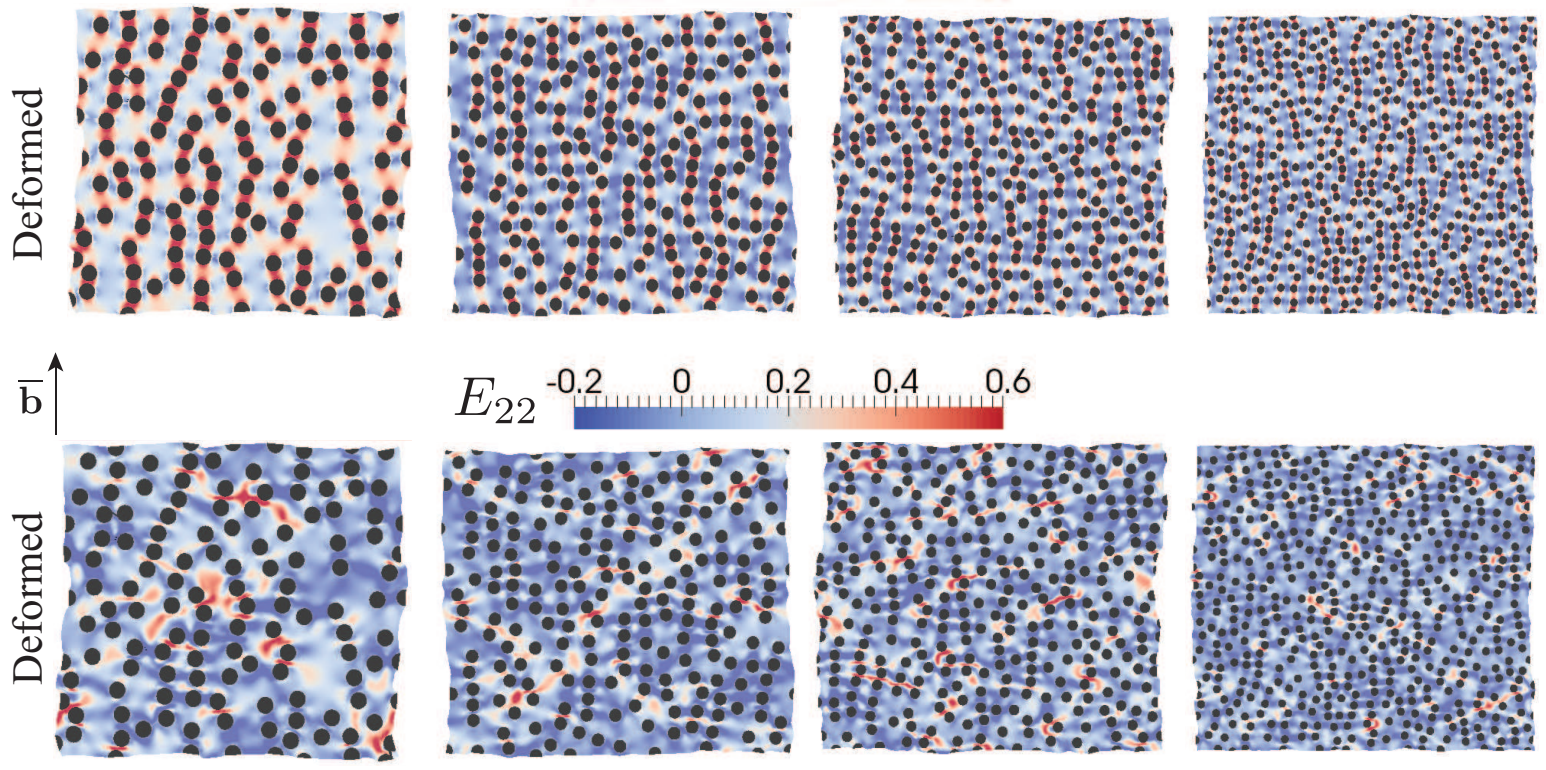

Figure 9: Deformed unit cells with isotropic distributions of particles and volume fraction $c=25$ vol\%. We show four unit cells with number of particles $N_{p}=120,200,300,500$. Contours of the normalized magnetic field $b_{2} / \mu_{0} m_{s}$ (top row) and of the Green-Lagrange strain $E_{22}$ (bottom row) in the direction of the applied magnetic field $\overline{\mathbf{b}}=\bar{b} \mathbf{e}_{2}$. The magnetic field contours indicate the chain formation and the interaction of the particles. Even though, the overall (average) strain in the unit cell is compressive (i.e., $\bar{E}_{22} \sim-0.05<0$ ), regions of very high tensile strain $E_{22}>0.6$ are observed. This is due to the significant re-organization of the particles locally to align with the applied magnetic field.

magnetization $\bar{m}_{2} / m_{s}$ and the average magnetostriction $\bar{\lambda}_{2}-1$. The average magnetization response exhibits a monotonic increase with the volume fraction in the context of isotropic distributions as shown in Fig. 10a. Focusing further on the case of $c=15 \mathrm{vol} \%$ in Fig. 10a, we observe that the magnetization saturates at the same value as in the previous case of a single elliptical particle (see Fig. 5a). This is a rather interesting result stating simply that the saturation magnetization of a MRE composite is independent of the microstructure (i.e., particle shape, distribution and orientation) but only depends upon the specific particle volume fraction. This observation is in complete agreement with the experimental results of Danas et al. (2012), which revealed exactly the same phenomenon in the case of particle chains which will be discussed in the following section. Furthermore, the present numerical results indicate that the average saturation magnetization, denoted here as $\bar{m}_{s}$, can be simply obtained by a simple rule of mixtures of the individual saturation magnetization of each phase, i.e.,

$$
\bar{m}_{s}=\sum_{r=1}^{N_{f}} c^{(r)} m_{s}^{(r)},
$$

where $N_{f}$ is the number of the phases and $c^{(r)}$ is the volume fractions of each phase. That is of course not true for the average magnetic susceptibility (i.e., the initial slope of the $\bar{m}-\bar{b}$ curve), which depends strongly upon the distribution effects as well as the particle shapes. Note that, in the case of two-phase materials, equation (5.3) can be used inversely to identify the "volume fraction" of the magnetic phase in a non-magnetic matrix, simply by measuring the overall magnetization of the composite. This is a rather useful result provided that we know the saturation magnetization of the magnetic phase. 
a)

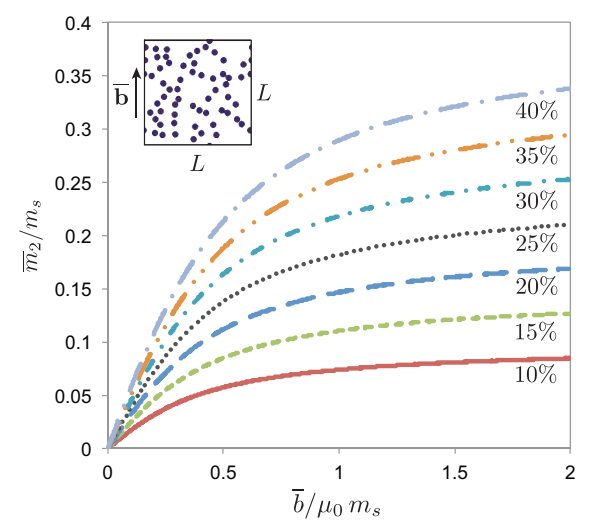

b)

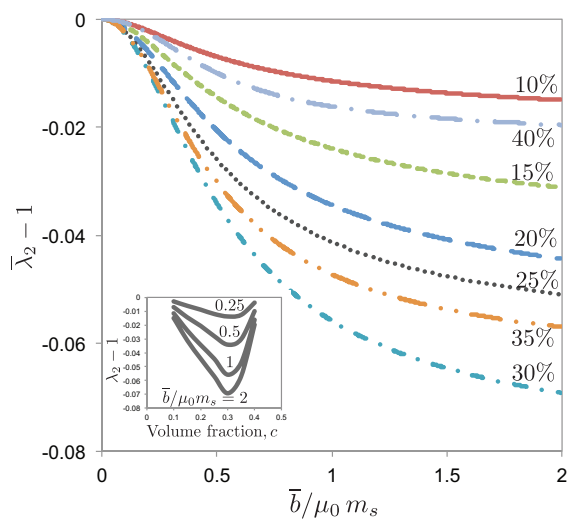

Figure 10: Macroscopic response of unstructured multi-particle periodic unit cells with external aspect ratio $w_{d}=1$ comprising circular rigid magnetizable inclusions randomly distributed in the unit cell for a volume fraction of $c=25 \mathrm{vol} \%$. The composite is subjected to a magnetic field in the $X_{2}$ direction and zero overall mechanical traction. (a) Average magnetization and (b) average magnetostriction as a function of the normalized average Eulerian magnetic field $\bar{b} / \mu_{0} m_{s}$ for various particle volume fractions $c=10,15,20,25,30,35,40 \mathrm{vol} \%$. The inset in part (b) shows the magnetostriction response as a function of the particle volume fraction $c$ for various values of the applied magnetic field $\bar{b} / \mu_{0} m_{s}$. A maximum magnetomechanical coupling is observed at volume fraction around $c \sim 30 \mathrm{vol} \%$.

In turn, the corresponding average magnetostriction, shown in Fig. 10b, is compressive and exhibits a non-monotonic dependence on the particle volume fraction $c$ with its maximum (in absolute value) lying at about $c=30 \mathrm{vol} \%$ (see also Diguet et al. (2010) but note that in their results the specimen effect is predominant). This is more clear by observing the inset in Fig. 10b, which shows the magnetostriction response as a function of the particle volume fraction $c$ for various values of the applied magnetic field $\bar{b} / \mu_{0} m_{s}$. A maximum magnetomechanical coupling is observed at volume fraction around $c \sim 30 \mathrm{vol} \%$. In the same figure, we also observe that the magnetomechanical coupling drops sharply with further increase of the volume fraction to $40 \mathrm{vol} \%$. The obtained results for the magnetostruction are in fact in agreement with the experiments and analysis carried out by Martin et al. (2006), who used approximate demagnetizing factors to take out the specimen shape effect.

\subsection{Idealized particle-chain microstructure}

In this section, we investigate the effect of particle-chain microstructures upon the effective response of the MRE unit cell. Such microstructures have been studied experimentally in the literature (see for instance Danas et al. (2012)) and lead to an anisotropic magnetomechanical response. In order to study such anisotropic effects at large magnetic fields we modify the material parameters used in the previous sections, as shown in Table 2. This is done to avoid significant mesh distortion present in such dense particle-packings in the particle-chain directions and thus reach higher magnetic fields without numerical convergence problems.

For comparison purposes, we consider two different unit cells in Fig. 11; one where the particles are perfectly aligned in a given direction denoted by the reference particle-chain orientation vector $\mathbf{N}$ (see Figs. 11a,b) and a second where the line connecting the two particle centers makes an angle of $10^{\circ}$ with respect to the vector $\mathbf{N}$ (see Figs. 11c,d). In the first case, the exterior unit cell has an aspect ratio $w_{d}=L_{2} / L_{1}=0.5$, while in the second case, $w_{d}=0.3^{5}$. In both cases the particle volume fraction is taken

\footnotetext{
${ }^{5}$ There are of course infinite number of choices for this imperfect particle chain but it is beyond the scope of the present analysis to carry out a parametric study with respect to the relevant geometric parameters that could describe the particle chains.
} 
to be equal to $c=20 \mathrm{vol} \%$. Then, following the work of Danas et al. (2012), one could define two possible configurations for the above defined unit cells. By always fixing the average applied magnetic field $\overline{\mathbf{b}}$ in direction $X_{2}$, one can consider the particle chains to be either parallel to the magnetic field, i.e., $\overline{\mathbf{b}} \| \mathbf{N}$ or perpendicular to it $(\overline{\mathbf{b}} \perp \mathbf{N})$.

\begin{tabular}{|c|c|c|c|c|c|c|c|c|}
\hline & $G(\mathrm{MPa})$ & $G^{\prime} / G$ & $E(\mathrm{MPa})$ & $\nu$ & $\chi$ & $\chi_{v}$ & $\mu_{0} m_{s}(\mathrm{~T})$ & $\mu_{0}\left(\mu \mathrm{N} \cdot \mathrm{A}^{-2}\right)$ \\
\hline Matrix & 0.5 & 1000 & 1.4995 & 0.4995 & 0.0 & 0.0 & - & $4 \pi 10^{-1}$ \\
\hline Particle & 500 & 1000 & 1499.5 & 0.4995 & 0.995 & 200 & 2 & $4 \pi 10^{-1}$ \\
\hline
\end{tabular}

Table 2: Material parameters for particle-chain calculations. The matrix values closely correspond to those for a natural rubber. The particle values describe carbonyl iron.

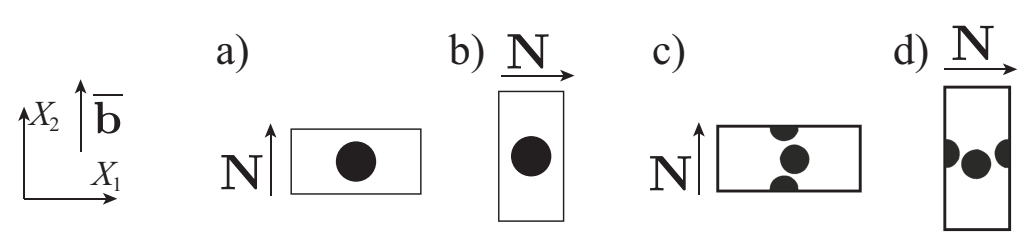

Figure 11: Sketch of perfect (a) and (b) and imperfect (c) and (d) particle-chain microstructures. The magnetic field is applied in the $X_{2}$ direction while the particle chains could be parallel to the applied magnetic field $(\overline{\mathbf{b}} \| \mathbf{N})$ or perpendicular to the magnetic field $(\overline{\mathbf{b}} \perp \mathbf{N})$.

a)

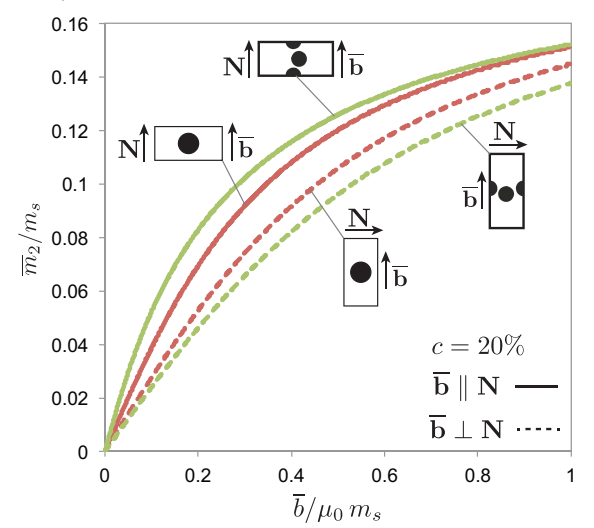

b)

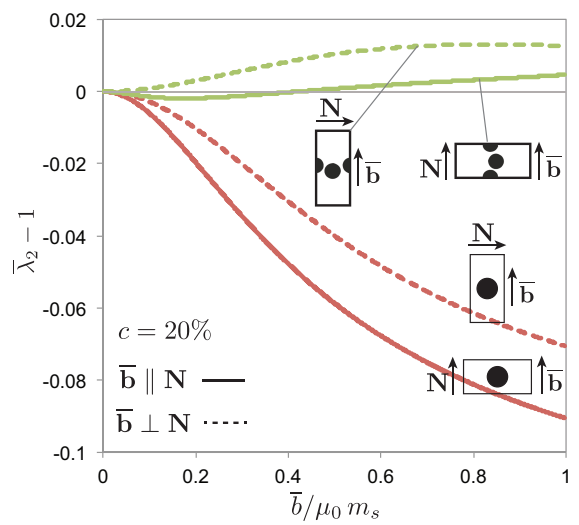

Figure 12: Macroscopic response of rectangular periodic unit cells comprising circular rigid magnetizable inclusions forming perfect or imperfect (wavy) chains whose orientation is denoted by the vector $\mathbf{N}$ at volume fraction $c=$ $20 \mathrm{vol} \%$. The composite is subjected to a magnetic field either along the chains, i.e., $\overline{\mathbf{b}} \| \mathbf{N}$ (parallel configuration) or perpendicular to the chains, i.e., $\overline{\mathbf{b}} \perp \mathbf{N}$ (perpendicular configuration). (a) Average magnetization and (b) average magnetostriction along the applied magnetic field as a function of the normalized average Eulerian magnetic field $\bar{b} / \mu_{0} m_{s}$.

In Fig. 12, we analyze the relative effect of the particle chain orientation with respect to the applied magnetic field. The parallel configurations are denoted with solid lines and the perpendicular ones with dashed lines. Specifically, in Fig. 12a, we observe that both perfect and imperfect parallel configurations lead to higher magnetization $\bar{m}_{2} / m_{s}$ slopes (i.e., effective magnetic susceptibility of the MRE composite) than the perpendicular ones. This result is in qualitative agreement with the experimental results of Danas et al. (2012). As stated earlier and again in agreement with experimental results the average saturation magnetization for the MRE is independent of the particle orientation and is the same for all configurations considered 
here. In turn, the corresponding magnetostriction estimates in Fig. 12b exhibit a much larger dependence on the particle chains configurations. While the perfect particle alignment leads to an overall compression of the MRE unit cell, the imperfect (wavy) particle chains lead to an overall extension of the unit cell (except for a small range for the imperfect parallel case). These results again are in qualitative agreement with the experiments of Danas et al. (2012) and recent numerical results by Kalina et al. (2016). Moreover, in the case of the wavy particle-chains, we observe that the perpendicular configuration $(\overline{\mathbf{b}} \perp \mathbf{N})$ leads to higher (positive) strains than the parallel one, which again is in qualitative agreement with the aforementioned experimental results. In addition to this, we also observe that the perfect particle-chains lead to much higher in amplitude (compressive) strains than the wavy ones. Thus, the present particle-chain microstructures probe successfully those experiments, at least in qualitative terms and shed some light on the design of MREs with particle chain microstructure. In addition, this last result shows the extremely sensitive dependence of the magnetostriction response upon the underlying microstructure. While the corresponding magnetization curves are very close in the case of perfectly and non-perfectly aligned particles (it suffices to compare the values for $\bar{b} / \mu_{0} m_{s}=0.5$ in Figs. 12a), the corresponding magnetostriction responses are very different. This implies that optimization of the MRE response by proper tuning of the microstructure, although possible, is a very tedious task and requires very careful analysis.

Finally, it is worth pointing out that in the wavy particle-chains, the shear strains are very high in the spacing between the particles. As in the case of the unstructured multi-particle unit cells, these high local shear strains lead to severe mesh distortion locally and subsequent cease of the calculations at relatively moderate magnetic fields.

\subsection{Effect of saturation function}

In this section, we investigate the effect upon magnetization and magnetostriction of the saturation functions defined in equations (2.11) and (2.12). For a better illustration of the effects, the shear modulus of the matrix phase is chosen to be higher, i.e., $G=1 \mathrm{MPa}$, so that we can reach higher magnetic fields without significant distortion of the mesh and cease of the calculations. We also chose to show results for two microstructures; the single particle square unit cell discussed in section 5.1 and the unstructured multiparticle unit cells presented in section 5.3. For brevity, we choose a volume fraction of $c=25 \mathrm{vol} \%$ for both microstructures. The material parameters used for the calculations are presented in Table 3.

\begin{tabular}{|c|c|c|c|c|c|c|c|c|}
\hline & $G(\mathrm{MPa})$ & $G^{\prime} / G$ & $E(\mathrm{MPa})$ & $\nu$ & $\chi$ & $\chi_{v}$ & $\mu_{0} m_{s}(\mathrm{~T})$ & $\mu_{0}\left(\mu \mathrm{N} \cdot \mathrm{A}^{-2}\right)$ \\
\hline Matrix & 1 & 100 & 2.99 & 0.495 & 0.0 & 0.0 & - & $4 \pi 10^{-1}$ \\
\hline Particle & 1000 & 100 & 2990 & 0.495 & 0.995 & 200 & 2 & $4 \pi 10^{-1}$ \\
\hline
\end{tabular}

Table 3: Material parameters to investigate the effect of the magnetization saturation function. The matrix values closely correspond to those for a natural rubber. The particle values describe carbonyl iron.

In Fig.13a, we observe that the magnetization response is initially the same for both saturation functions used for the particle, i.e., (2.11) and (2.12) as well as for both microstructures. For this volume fraction, $c=25 \mathrm{vol} \%$, the single particle unit cell and the unstructured multi-particle unit cells lead to the same initial effective susceptibility. In turn, the response diverges at larger magnetic fields where the saturation response becomes predominant. In that case, again the microstructure seems to have a weak effect on magnetization. The Langevin function (2.11) leads to much slower saturation response than the "tanh" saturation function (2.12). That is already visible in Fig.2, where we have simply plotted the derivatives of these functions.

The effect of the saturation functions (2.11) and (2.12) upon magnetostriction is much stronger than that for the magnetization response, as observed in Fig. 13b. The effect of the microstructure is also very important. It is easily seen that the "tanh" function (2.12) leads to higher magnetostriction response for both microstructures by comparison to the Langevin function (2.11) at moderate and large magnetic fields. At very small magnetic fields the saturation function has only minor effects. The magnetostriction response for the "tanh" function (2.12) saturates much faster, while the one for the Langevin function (2.11) is much slower, especially in the case of the single-particle unit cell. Finally, it is interesting to note that single-particle unit cell leads to significantly larger in absolute value (compressive) magnetostriction than 
a)

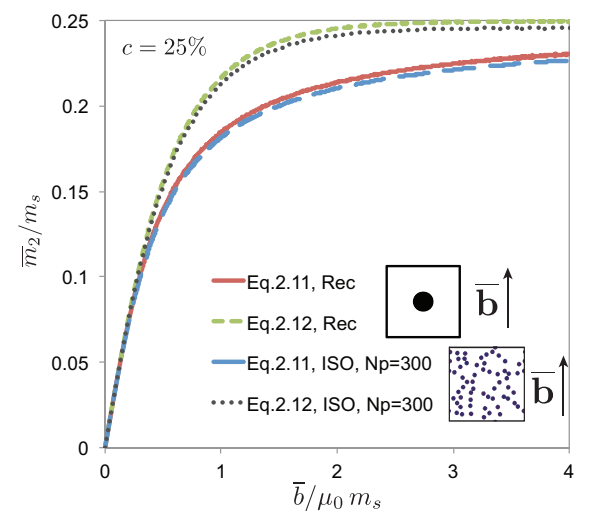

b)

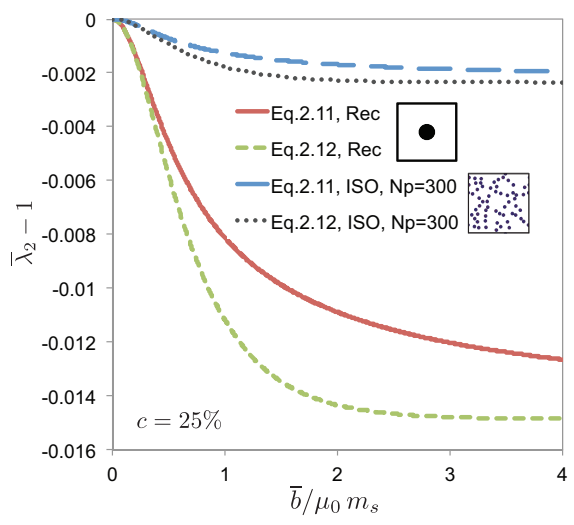

Figure 13: Macroscopic response of a single circular-particle square unit cell and unstructured multi-particle periodic unit cells at volume fraction $c=25 \mathrm{vol} \%$. The composite is subjected to a magnetic field in the $X_{2}$ direction and zero overall mechanical traction. (a) Average magnetization and (b) average magnetostriction as a function of the normalized average Eulerian magnetic field $\bar{b} / \mu_{0} m_{s}$ for the two saturation functions described in equations (2.11) (Langevin model) and (2.12) ("tanh" model).

the unstructured multi-particle unit cells, even if at small fields the magnetization response is almost the same.

\section{Results : Active auxetic and chiral microstructures}

In this section, we use the developed framework to probe microstructures that exhibit auxetic response in purely mechanical loads but can now be triggered and dynamically controlled by magnetomechanical loads. In order to analyze such microstructures, we use the same energy functions defined in (5.1). Specifically, we deal with three-phase unit cells where the matrix phase is a non-magnetic polymer/elastomer, the particles are magnetically soft but mechanically stiff, while the third phase is void/air. The above choice of materials constitutes a periodic unit cell with very large contrast between the phases (the maximum contrast is the one between the void/air and the particle) both mechanically and magnetically. This renders the numerical simulation rather sensitive in terms of convergence.

\subsection{Three-phase auxetic unit cells: particle, matrix and voids}

In this section, we study a three-phase unit cell with voids distributed in a square array, while particles forming a perfect chain along direction $X_{2}$. The void volume fraction is $45 \mathrm{vol} \%$ and the particle volume fraction is $15 \mathrm{vol} \%$. The voids are intentionally designed to have an initially elliptical shape whose longest axis is in an alternating orientation of $90^{\circ}$ and $0^{\circ}$, as shown in Fig. 14. The results presented in this section, make use of the material constants presented in Table 4. This choice allows for a satisfactory convergence of the simulations for rather large strains, especially at the void phase which is also meshed to resolve the magnetic Maxwell stresses therein.

\begin{tabular}{|c|c|c|c|c|c|c|c|c|}
\hline & $G(\mathrm{MPa})$ & $G^{\prime} / G$ & $E(\mathrm{MPa})$ & $\nu$ & $\chi$ & $\chi_{v}$ & $\mu_{0} m_{s}(\mathrm{~T})$ & $\mu_{0}\left(\mu \mathrm{N} \cdot \mathrm{A}^{-2}\right)$ \\
\hline Matrix & 0.05 & 100 & 0.1495 & 0.495 & 0.0 & 0.0 & - & $4 \pi 10^{-1}$ \\
\hline Particle & 50 & 100 & 149.5 & 0.495 & 0.995 & 200 & 2 & $4 \pi 10^{-1}$ \\
\hline Voids & $5 . \mathrm{e}-4$ & 1 & $1.25 \mathrm{e}-3$ & 0.25 & 0.0 & 0.0 & - & $4 \pi 10^{-1}$ \\
\hline
\end{tabular}

Table 4: Material parameters corresponding to a silicon matrix, a mechanically hard magnetic particle and air. 
The unit cell of Fig. 14 can lead to an apparent "negative Poisson" effect at the macrostructural level when subjected to uniaxial compression loads (Bertoldi et al., 2010). In the present case of the periodic unit cell, the addition of a particle-chain pattern, which is magnetically active allows to induce such compressive loads via an externally applied magnetic field either along or perpendicular to the particle chain. As the particles attract or repel each other, an overall compression is induced in the direction of the applied magnetic field leading to interesting deformation patterns.

$$
\bar{b} / \mu_{0} m_{s}=0 \quad \bar{b} / \mu_{0} m_{s}=0.25 \quad \bar{b} / \mu_{0} m_{s}=0.45
$$
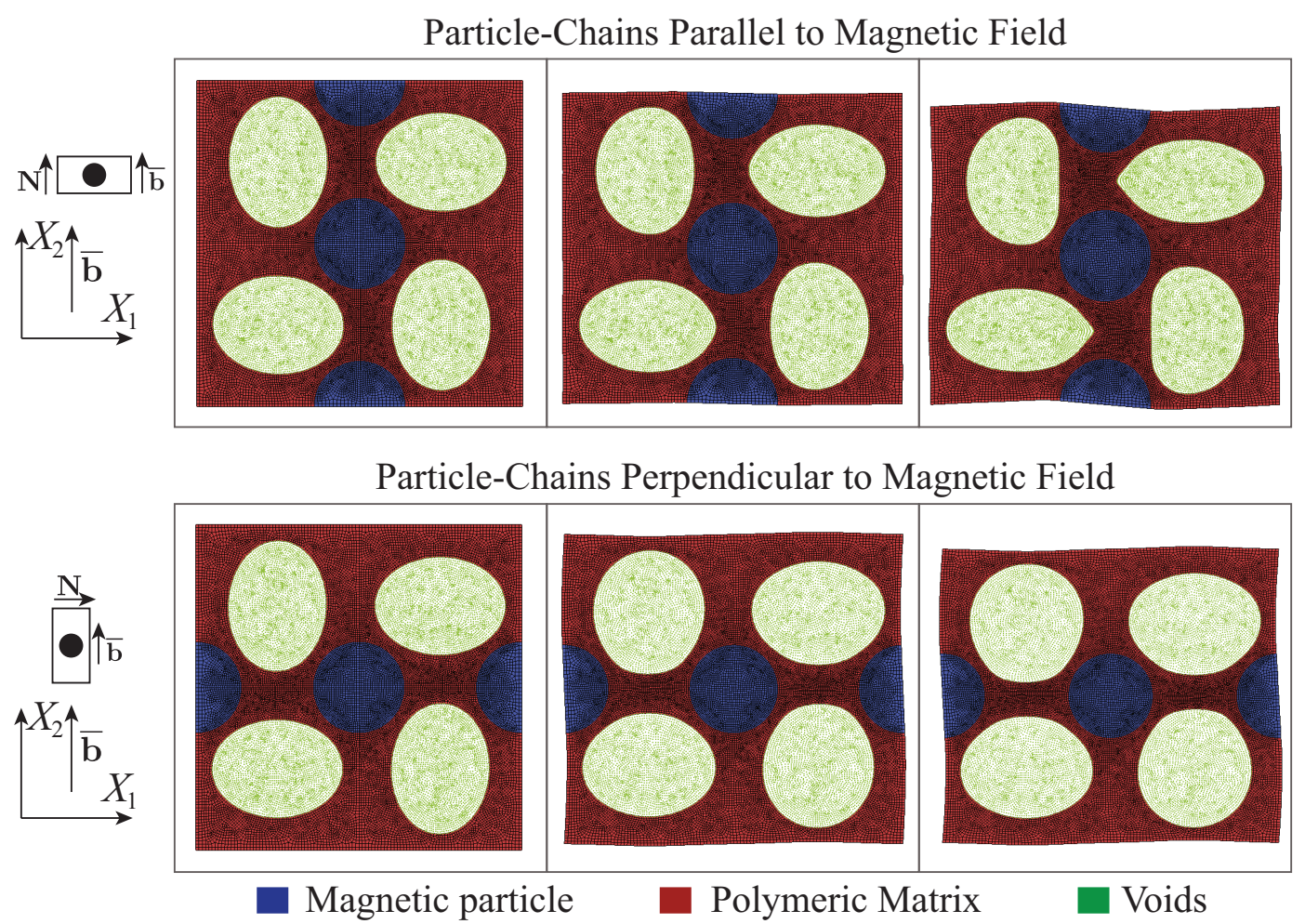

Figure 14: Deformed unit cells comprising three phases: magnetic particles (blue), non-magnetic polymeric matrix (red) and voids (green). The particles are distributed in a chains while the voids are ellipsoidal in shape. The voids have been drawn such that they reproduce an auxetic response according to earlier work in the literature. In the top row the particle chains are parallel to the applied magnetic field $\bar{b}$ in $X_{2}$ direction. At the bottom row, the particle chains (sketched using the unit vector $\mathbf{N}$ ) are perpendicular to the applied magnetic field $\bar{b}$ in $X_{2}$ direction. Under application of a magnetic field the particles attract and thus lead to overall contraction of the unit cell.

As shown in Fig. 14, by gradual increase of the applied magnetic field, in the case where the particles are parallel to $\overline{\mathbf{b}}$, they attract each other and thus induce a compressive overall response. In turn, when the particle-chains are set perpendicular to the applied magnetic field, they repel each other and thus an overall compressive response is induced again in the direction of the applied magnetic field, similar to the observations in the context of perfect particle-chain microstructures of the previous section. Note that in the parallel configuration case, a small tip starts forming in two (upper right and lower left) out of the four voids. In the perpendicular case, the void shapes evolve differently from those in the parallel case. This difference can mainly be attributed to the presence of the stiff particle-chains and the induced anisotropic response of the surrounding polymer/non-magnetic phase.

The quantitative response of the above-described unit cell is described in Fig. 15. In part (a), we show the magnetization response which shows very similar trends with the ones obtained for the single particle-chain unit cell in Section 5.4. In the case of the parallel configuration (i.e., magnetic particle-chain parallel to the 
a)

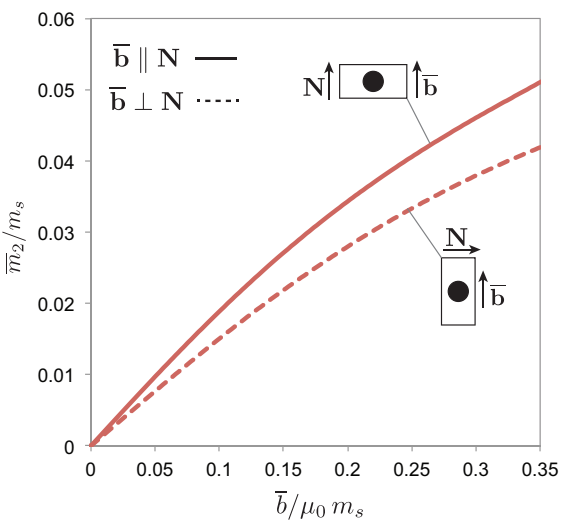

b)

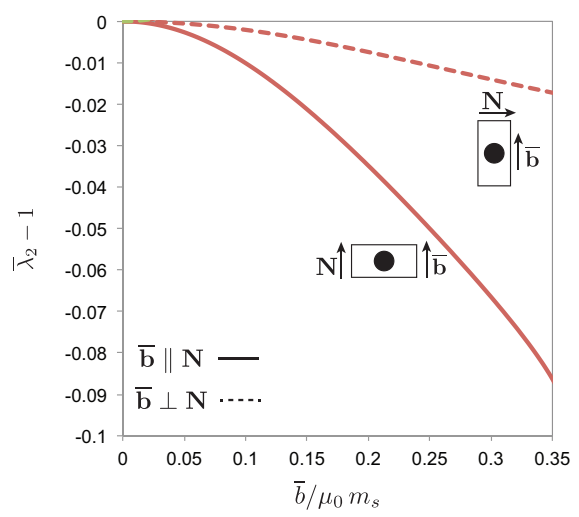

Figure 15: Macroscopic response of the three phase unit cell comprising magnetic particles, polymeric matrix (nonmagnetic) and voids. The particles are distributed in a chains while the voids are ellipsoidal in shape. The composite is subjected to a magnetic field either along the chains, i.e., $\overline{\mathbf{b}} \| \mathbf{N}$ (parallel configuration) or perpendicular to the chains, i.e., $\overline{\mathbf{b}} \perp \mathbf{N}$ (perpendicular configuration). (a) Average magnetization and (b) average magnetostriction along the applied magnetic field as a function of the normalized average Eulerian magnetic field $\bar{b} / \mu_{0} m_{s}$.

applied field) the magnetization (and hence material susceptibility) is higher than in the perpendicular one. In turn, in Fig. 15b, we show the magnetostriction induced in the direction of the applied magnetic field. Again, as already observed in the context of Fig. 12b for the two-phase particle-chain unit cell, the parallel configuration leads to larger compressive strains along the applied magnetic field than the perpendicular one.

\subsection{Three-phase auxetic, chiral unit cells: particle, matrix and air}

In this section, the proposed unit cell uses geometrical chirality and particle shapes to induce negative and positive swelling. Chirality is the notion of a material exhibiting preferential rotational (chiral) directions upon application of mechanical or other fields, such as magnetic ones in the present case. It is of importance in natural processes and recently scientists try to take advantage of it in man made materials. While in the present case, one can design the unit cell to produce well controlled directional instabilities and pattern formations, we choose instead an entirely stable configuration by placing the elliptical particle at an angle that induces a pure rotation without a bifurcation.

As we have shown in the context of Fig. 5, elliptical (or more generally elongated) magnetic particles (or materials) tend to rotate under the application of a magnetic field if it is applied perpendicular to the long axis of the ellipse. This is due to the compass effect or in physics terms due to the minimization of the demagnetization energy of the particle. Using this property, one may propose auxetic structures taking advantage of the particle rotation in tri/tetra/hexa-chiral microstructures. The potential micro- or meso-structures are numerous so for illustration purposes, we show in Fig. 16 one such possible unit cell. It comprises an elliptical magnetic particle (blue), non-magnetic polymeric matrix (red) in form of strips and surrounding air (green). The longest axis of the elliptical particle forms an angle of $10^{\circ}$ with respect to the $X_{1}$ axis. The specific details of the structure (i.e., fillets and size of strips) are too complex to describe here and probably unnecessary for the presentation of the results. The results presented in this section, make use of the material constants presented in Table 5. This choice allows for realistic material parameters as well as satisfactory convergence of the simulations for rather large strains in this case of very high volume fraction of air and large contrast among the phases. Again the air is fully meshed in order to resolve the Maxwell stresses therein.

More specifically, in the top-row of Fig. 16, the elastic (non-magnetic strips) are designed such that, under application of the magnetic field and subsequent rotation of the magnetic particle, the unit cell shrinks in 


\begin{tabular}{|c|c|c|c|c|c|c|c|c|}
\hline & $G(\mathrm{MPa})$ & $G^{\prime} / G$ & $E(\mathrm{MPa})$ & $\nu$ & $\chi$ & $\chi_{v}$ & $\mu_{0} m_{s}(\mathrm{~T})$ & $\mu_{0}\left(\mu \mathrm{N} \cdot \mathrm{A}^{-2}\right)$ \\
\hline Matrix & 1.0 & 100 & 2.99 & 0.495 & 0.0 & 0.0 & - & $4 \pi 10^{-1}$ \\
\hline Particle & 500 & 100 & 1495 & 0.495 & 0.995 & 200 & 2 & $4 \pi 10^{-1}$ \\
\hline Air & $5 . \mathrm{e}-3$ & 1 & $1.25 \mathrm{e}-2$ & 0.25 & 0.0 & 0.0 & - & $4 \pi 10^{-1}$ \\
\hline
\end{tabular}

Table 5: Material parameters corresponding to a natural rubber matrix phase, mechanically hard magnetic particle and air.

Negative swelling, $\bar{J}<1$

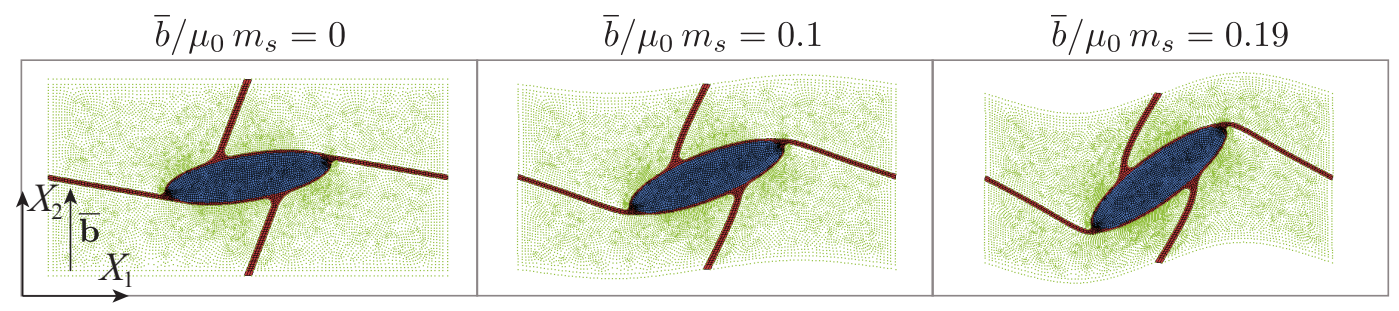

Positive swelling, $\bar{J}>1$

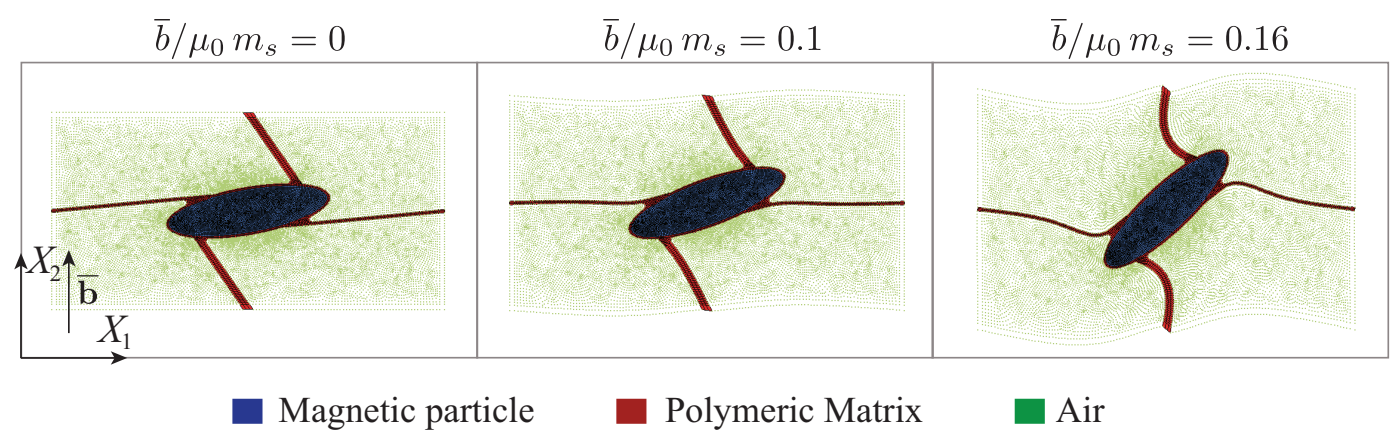

Figure 16: Deformed unit cells comprising three phases: an elliptical magnetic particle (blue), non-magnetic polymeric matrix (red) in form of strips and surrounding air (green). The elliptical particle forms an angle of $10^{\circ}$ with respect to the $X_{1}$ axis. The magnetic field $\overline{\mathbf{b}}$ is applied along the $X_{2}$ direction and induces a rotation of the elliptical particle. This rotation in turn results in an overall auxetic response of the unit cell. At the top row, the unit cell is designed such that upon application of the magnetic field along $X_{2}$ direction to produce negative swelling, i.e., $\bar{J}<1$. At the bottom row the unit cell is designed to produce positive swelling, i.e., $\bar{J}>1$. The unit cell in the bottom row could be thought as one obtained by mirroring the matrix strips with respect to a line forming a $10^{\circ}$ angle with respect to the $X_{1}$ axis, i.e., similar to that of the long axis of the ellipse. These unit cells can lead to an overall auxetic response.

both directions leading to an overall negative swelling (or volume reduction) measured by the average third invariant, i.e., $\bar{J}<1$. The reduction of the volume is directly observable in the upper row of Fig. 16. Next, at the bottom row of Fig. 16, we reverse the chirality of the unit cell by mirroring the matrix strips with respect to a line forming a $10^{\circ}$ angle with respect to the $X_{1}$ axis, i.e., similar to that of the long axis of the ellipse. This unit cell, then, leads to positive swelling (or volume increase) under application of the magnetic field. In both cases the magnetic field is applied in the $X_{2}$ direction which is almost perpendicular to the long axis of the ellipse. This, in turn, induces torques and rotation of the ellipse. One could also observe in Fig. 16 that in the case of positive swelling the matrix strips tend to buckle since they are subjected to compressive loads. That is not the case in the negative swelling unit cell of the top-row where all strips are under tensile loads.

The quantitative response of the previously described unit cell is shown in Fig. 17. In part (a) we show 
a)

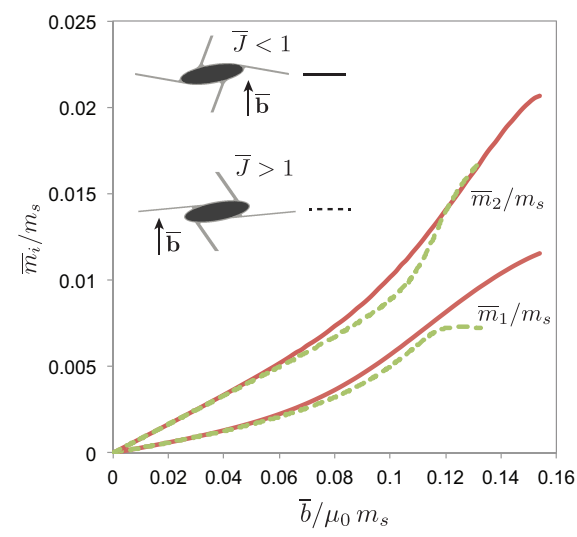

b)

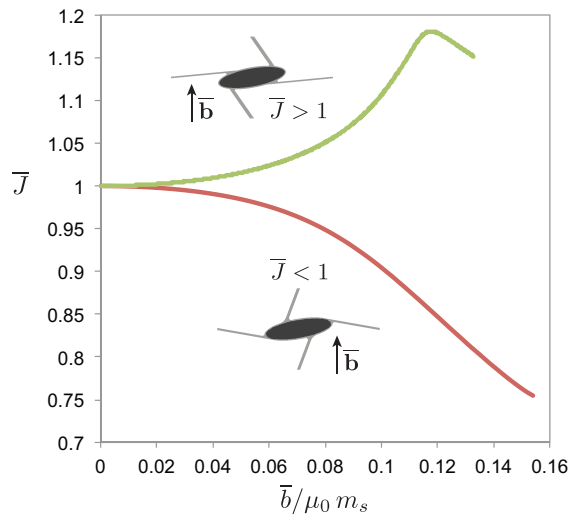

Figure 17: Macroscopic response of the three phase unit cell comprising an elliptical magnetic particle, polymeric matrix (non-magnetic) in form of strips and surrounding air. The elliptical particle forms an angle of $10^{\circ}$ with respect to the $X_{1}$ axis, while the magnetic field $\overline{\mathbf{b}}$ is applied along the $X_{2}$ direction. (a) Average magnetization along, $\bar{m}_{2} / m_{s}$ and perpendicular, $\bar{m}_{1} / m_{s}$, to the applied magnetic field and (b) average volume change $\bar{J}$ as a function of the normalized average Eulerian magnetic field $\bar{b} / \mu_{0} m_{s}$. The continuous lines describe the negative swelling unit cell and the dashed ones the positive swelling unit cell.

the corresponding average magnetization along, $\bar{m}_{2} / m_{s}$, and perpendicular, $\bar{m}_{1} / m_{s}$, to the overall applied magnetic field while in part (b) we trace the average volume change in the unit cell by showing $\bar{J}=\operatorname{det} \overline{\mathbf{F}}$ as a function of the applied magnetic field. Rather surprisingly, in Fig. 17a the magnetization has a very small linear regime and then increases in a nonlinear manner (becomes convex) until reaching saturation (mostly visible in the negative swelling unit cell or red curve) becoming concave again. Perhaps it is relevant to mention at this point that a similar nonlinear (convex) increase of the electric polarization has been recently obtained by Cohen et al. (2016). More interestingly, one can observe that such a nonlinearity is not present in the case of an elliptical particle in a polymeric matrix as shown in Fig. 5. Instead, this effect seems to be present in the auxetic unit cells of Fig. 17, which show significant negative or positive swelling. Moreover, the two unit cells exhibit almost the same magnetization response, indicating that the observed nonlinearity in the magnetization response is mainly driven by the particle rotation as well as the presence of the air, which is the same in these two cases. A second interesting observation in the context of Fig. 17a is that the transverse component $\bar{m}_{1}$ is also positive and increasing, contrary to the case of the elliptical particle in a matrix shown in the inset of Fig. 5a. This rather non-intuitive response is strongly linked to the swelling of the proposed unit cell.

Finally, in Fig. 17b, we show $\bar{J}$ as a function of the applied magnetic field. Significant levels of negative $(\sim 25 \%)$ and positive $(\sim 20 \%)$ swelling are obtained with the proposed unit cells before saturation. The observed values are obtained at rather small values of the magnetic fields and thus make such unit cells very promising for real applications. The numerical simulations cease when the elements used to simulate the surrounding air become highly distorted.

\section{Concluding Discussion}

In this work, we have investigated in depth the pure effect of the underlying microstructure upon the coupled response of a magnetoelastic composite by decoupling it from the structural/geometrical shape effects present in a magnetomechanical experiment (see Fig. 1a and Fig. 3). This approach allows us to better understand the pure contribution of the material response on magnetostriction, since in a standard boundary value problem macroscopic geometry and microscopic material effects are strongly coupled. As a result the observed magnetostriction could be easily misinterpreted if the contributions of the macroscopic geometry and the material are not properly separated. 
We have also clarified the differences between a coupled magnetomechanical analysis in which one applies a Eulerian (current) magnetic field and an electroactive one where the Lagrangian (reference) electric field is usually applied. In order to achieve such a task, we have presented an augmented variational principle to analyze the homogenized magnetoelastic response of periodic representative volume elements (RVEs). The augmented variational principle derives directly from the one of Dorfmann and Ogden (2003), which is more than enough to describe magnetoelastic boundary value problems but requires modification in the present context of magnetoelastic homogenization. The need for its modification arises in the case of periodic unit cells where the surrounding of the RVE medium is not directly modeled; instead periodicity conditions are applied at the boundary of the unit cells. For this, one needs to include two additional terms in the original variational formulation; the first serves to impose continuity of the average Maxwell stress arising due to the presence of the magnetoelastic medium itself that surrounds the periodic unit cell (see equation (4.1)) and the second term allows us to apply the average Eulerian magnetic field instead of the Lagrangian one (see equation $(4.2)$ ).

Subsequently, the proposed augmented variational principle has been used to analyze the effect of particle distributions, particle shape and particle orientation. Specifically, the effect of the relative orientation of the long axis of the ellipse with respect to the direction of the applied magnetic field has been studied in detail. As a general observation, the long axis of the elliptical particle tends to align with the applied magnetic field (which is the well known compass effect). As a result of this particle rotation, the magnetostriction response has been found to be a strongly non-monotonic function of the orientation angle, which can lead to either extension or compression of the RVE. In addition, when the ellipse long axis is exactly perpendicular to the direction of the applied magnetic field, we observe the onset of a bifurcation exactly at the critical point that the particle attempts to rotate. This rotational (torque) effect has been exploited recently at a macroscopic scale in a magnetorheological film/substrate structure to induce magnetomechanically driven wrinkles (Danas and Triantafyllidis, 2014), in a rotating magnetic rod Gerbal et al. (2015) and in a single magnetic particle-chain (Huang et al., 2016).

A number of important results have also been obtained in the context of unstructured isotropic microstructures, which were obtained by standard random adsorption algorithms. An important step in analyzing such isotropic microstructures has been a convergence study of the representativity of the proposed unit cells. Rather interestingly we have observed that while for the pure average magnetization response one needs 60 particles to obtain a representative volume element that is not the case for the magnetostriction response, which requires at least 300 particles. This behavior appears to be partially related to the fact that the magnetostriction response is ab initio a nonlinear function (initially quadratic) of the applied magnetic field. This non-linearity, albeit a weak one, requires a large number of particles in the unit cell in order to reach representativity. Other possible reasons for this high number of particles needed for representativity of the unit cell are also the prescribed boundary conditions, i.e., the application of the Eulerian magnetic field and not the Lagrangian one as well as the rather low shear modulus of the matrix phase used in those calculations. Another important result in the context of isotropic microstructures is related to the overall contraction of the unit cell in the direction of the applied magnetic field. At first this might seem contradictory to existing experiments. Nonetheless, the present analysis considers the pure effect of the microstructure at the RVE scale free of any effects of the specimen shape present in a real experiment. This result is in fact in agreement with the experiments and analysis carried out by Martin et al. (2006), who used approximate demagnetizing factors to take out the specimen shape effect.

An additional interesting and rather practical observation in the context of all microstructures analyzed here has been the fact that the average saturation magnetization of the unit cell is independent of the particle orientation or distribution effects. Instead, it is a simple function of the particle volume fraction and may be obtained by a simple rule of mixtures of the individual saturation magnetization of the phases. That is of course not true for the average magnetic susceptibility (i.e., the initial slope of the $\bar{m}-\bar{b}$ curve), which depends upon the distribution effects, the particle shapes and more generally on the entire unit cell morphology.

Next, we have studied the effect of particle-chains by considering two idealized unit cells; one with "wavy" particle chain distribution and one with perfectly aligned particle chains. In the case of the wavy particle-chains, we have observed that the perpendicular configuration, i.e., the case where the magnetic 
field is applied perpendicular to the particle chains leads to higher (positive) strains than in the parallel one. This result is in qualitative agreement with the experimental results of Danas et al. (2012). In contrast, the perfect particle-chain unit cell has exhibited a pure compressive strain in the direction of the applied field, which in turn was much higher in amplitude than that in the wavy particle-chain unit cell.

The final section of this study has been devoted to novel three-phase unit cells that can exhibit auxetic response at the macroscale, but can now be triggered and dynamically controlled by properly combined magnetomechanical loads. These unit cells comprise three phases; a non-magnetic matrix phase, a magnetic particle phase and void/air. The unit cells proposed in this study have been inspired by corresponding unit cells in the purely mechanical context and are properly extended here in the context of coupled magnetoelasticity (and obviously can be used also in the context of coupled electroelasticity). The first unit cell makes use of the magnetic particle attraction under application of a magnetic field and can effectively lead to an overall compression which in turn can lead to an interchanging void pattern (Michel et al., 2007). The resulting pattern has been shown to lead to an apparent negative Poisson ratio (Bertoldi et al., 2010), which in turn can potentially lead to acoustic band gaps and interesting metamaterial properties (Prall and Lakes, 1997; Spadoni et al., 2009; Shan et al., 2014). The second unit cell uses the chirality of the microstructure and the rotation of an elliptical magnetic particle to induce large volume changes. These volume changes can lead to either negative or positive swelling depending on the sign of the chirality of the unit cell with respect to the direction of the applied magnetic field. From the practical point of view, it is worth noting that these fast and large volume changes (in the order of $\pm 25 \%$ ) can be achieved with relatively small magnetic fields $(\sim 0.1 T)$.

We conclude this discussion by noting that most of the microstructures studied in the present work are experimentally realizable (e.g., particle chains, isotropic and auxetic microstructures) and thus the present analysis provides the means to understand the underlying micro-deformation mechanisms and interactions in the context of soft magnetoelastic solids. Nevertheless, a number of additional effects that could be of importance at an experimental level, such as particle interfaces due to particle treatment, debonding and viscosity, as well as instabilities (Danas and Triantafyllidis, 2014; Wang et al., 2016) have not been considered. Moreover, we have only studied idealized particle-chain microstructures, since we do not know exactly how to obtain realistic particle-chains, which are of great practical importance in experiments. One possible way would be to simulate approximately the fabrication process, i.e., the particle-chain creation. Work along these lines is underway.

\section{Acknowledgments}

The author would like to thank N. Triantafyllidis for fruitful discussions and acknowledge support of the European Research Council (ERC) under the European Union's Horizon 2020 research and innovation program (grant agreement No 636903 - MAGNETO).

\section{A. A class of equivalent variational formulations}

In this section, we show that the variational formulation of Kankanala and Triantafyllidis (2004) is exactly equivalent to the augmented variational formulation (4.3), provided that one uses equivalent in the sense of partial Legendre-Fenchel transform energy functions for the materials at hand. In order to show this equivalence, we use the results of Bustamante et al. (2009). For clarity in our demonstration, we recall some of the definitions in that reference. We begin by defining the energy function $\widehat{\psi}^{(r)}(\mathbf{F}, \mathbf{h})$ via the partial Legendre transform with respect to $\mathbf{m}$

$$
\rho_{0}^{(r)} \widehat{\psi}^{(r)}(\mathbf{F}, \mathbf{h})=\rho_{0}^{(r)} \psi^{(r)}(\mathbf{F}, \mathbf{m})-J \mu_{0} \mathbf{m} \cdot \mathbf{h} .
$$

Next, one can define the total potential $\widehat{W}(\mathbf{F}, \mathbf{h})$ by

$$
\widehat{W}^{(r)}(\mathbf{F}, \mathbf{h})=\rho_{0}^{(r)} \widehat{\psi}^{(r)}(\mathbf{F}, \mathbf{h})-\frac{J \mu_{0}}{2} \mathbf{h} \cdot \mathbf{h}
$$


such that $W^{(r)}(\mathbf{F}, \mathbf{B})=w^{(r)}(\mathbf{F}, \mathbf{b})($ c.f. $(2.7))$ is obtained by the partial Legendre transform

$$
W^{(r)}(\mathbf{F}, \mathbf{B})=w^{(r)}(\mathbf{F}, \mathbf{b})=\widehat{W}^{(r)}(\mathbf{F}, \mathbf{h})+J \mathbf{b} \cdot \mathbf{h} .
$$

Combination of equations (A.1)-(A.3) together with relation (2.1) and definition (2.7) leads to

$$
\begin{aligned}
W^{(r)}(\mathbf{F}, \mathbf{B}) & =w^{(r)}(\mathbf{F}, \mathbf{b})= \\
& =\rho_{0}^{(r)} \Phi^{(r)}(\mathbf{F}, \mathbf{B})+\frac{1}{2 \mu_{0} J} \mathbf{F} \mathbf{B} \cdot \mathbf{F} \mathbf{B}= \\
& =\rho_{0}^{(r)} \phi^{(r)}(\mathbf{F}, \mathbf{b})+\frac{J}{2 \mu_{0}} \mathbf{b} \cdot \mathbf{b}= \\
& =\rho_{0}^{(r)} \psi^{(r)}(\mathbf{F}, \mathbf{m})-J \mu_{0} \mathbf{h} \cdot \mathbf{m}-\frac{J \mu_{0}}{2} \mathbf{h} \cdot \mathbf{h}+J \mathbf{b} \cdot \mathbf{h}= \\
& =\rho_{0}^{(r)} \psi^{(r)}(\mathbf{F}, \mathbf{m})-J \mu_{0} \mathbf{h} \cdot \mathbf{m}-\frac{J \mu_{0}}{2} \mathbf{h} \cdot \mathbf{h}+J \mu_{0} \mathbf{h} \cdot \mathbf{h}+J \mu_{0} \mathbf{m} \cdot \mathbf{h}= \\
& =\rho_{0}^{(r)} \psi^{(r)}(\mathbf{F}, \mathbf{m})+\frac{J \mu_{0}}{2} \mathbf{h} \cdot \mathbf{h}= \\
& =W_{m}^{(r)}(\mathbf{F}, \mathbf{m}, \mathbf{h}) .
\end{aligned}
$$

In this expression, we recall the definition $(2.8)$, i.e., $\Phi^{(r)}(\mathbf{F}, \mathbf{B})=\phi^{(r)}(\mathbf{F}, \mathbf{b})$. The above expression simply implies that

$$
\rho_{0}^{(r)} \psi^{(r)}(\mathbf{F}, \mathbf{m})=\rho_{0}^{(r)} \phi^{(r)}(\mathbf{F}, \mathbf{b}(\mathbf{m}))+\frac{J}{2 \mu_{0}} \mathbf{b}(\mathbf{m}) \cdot \mathbf{b}(\mathbf{m})-\frac{J \mu_{0}}{2} \mathbf{h}(\mathbf{m}) \cdot \mathbf{h}(\mathbf{m}),
$$

where the dependence of $\mathbf{b}$ and $\mathbf{h}$ on $\mathbf{m}$ has been explicitly noted in this last expression.

Illustrative example. It is perhaps useful at this point to present an example of the evaluation of $\psi^{(r)}$ if one is given $\phi^{(r)}$. For this, we will use definition (2.8) together with the simple quadratic function (2.15) such that

$$
\rho_{0}^{(r)} \phi^{(r)}(\mathbf{F}, \mathbf{b})=\rho_{0}^{(r)} \phi_{m e c h}^{(r)}(\mathbf{F})-\frac{J \chi^{(r)}}{2 \mu_{0}} \mathbf{b} \cdot \mathbf{b} .
$$

For this quadratic function, solving (2.16) for $\mathbf{b}$ and $\mathbf{h}$ as a function of $\mathbf{m}$, one obtains that

$$
\mathbf{b}(\mathbf{m})=\frac{\mu_{0}}{\chi^{(r)}} \mathbf{m}, \quad \mathbf{h}(\mathbf{m})=\frac{1-\chi^{(r)}}{\chi^{(r)}} \mathbf{m}, \quad \chi^{(r)}>0 .
$$

Then, substitution of (A.6) and (A.7) in (A.5) gives

$$
\rho_{0}^{(r)} \psi^{(r)}(\mathbf{F}, \mathbf{m})=\rho_{0}^{(r)} \phi_{m e c h}^{(r)}(\mathbf{F})+\frac{J \mu_{0}\left(1-\chi^{(r)}\right)}{2 \chi^{(r)}} \mathbf{m} \cdot \mathbf{m}, \quad \chi^{(r)}>0 .
$$

The above-described demonstrations are unique only if $\psi^{(r)}$ are convex and $\phi^{(r)}$ concave functions of $\mathbf{m}$ and $\mathbf{b}$, respectively. That is usually the case in magnetoelastic materials and particularly those proposed in equations (2.11) and (2.12).

Of course the previously-described procedure of getting $\psi^{(r)}$ from $\phi^{(r)}$ and vice versa directly implies that depending on the different forms of the functions that have been used in the literature for a number of them inverting the relations between $\mathbf{b}-\mathbf{m}$ and $\mathbf{h}-\mathbf{m}$ might not be analytically tractable, especially when the $\mathbf{b}-\mathbf{m}$ relation is nonlinear, as for example in the case of the Langevin function (2.11).. This, however, does not mean that the two functions are not equivalent in the general sense.

Next, we introduce a number of relations necessary for the subsequent analysis. By using the definition of the periodic boundary conditions in (3.2) together with (3.3), one can readily decompose the magnetic field $\mathbf{b}$ in an average one $\overline{\mathbf{b}}$ and a periodic perturbation $\widetilde{\mathbf{b}}$ (or equivalently its Lagrangian part $\widetilde{\mathbf{B}}$ ), such that

$$
\mathbf{b}=\overline{\mathbf{b}}+\widetilde{\mathbf{b}}, \quad \frac{1}{|\mathbf{q} \mathcal{V} \#|} \int_{|\mathbf{q} \mathcal{V} \#|} \widetilde{\mathbf{b}}(\mathbf{x}) \mathrm{d} v=\mathbf{0}, \quad \widetilde{\mathbf{b}}=\frac{1}{\bar{J}} \overline{\mathbf{F}} \widetilde{\mathbf{B}}, \quad \mathbf{B}=\overline{\mathbf{B}}+\widetilde{\mathbf{B}}
$$


These relations will be extensively used in the following, while it is worth noting that the perturbation Lagrangian magnetic field is simply $\widetilde{\mathbf{B}}=\operatorname{Curl} \widetilde{\mathbf{A}}$ (see equation (3.2)).

\section{A.1. Replace independent variable $\boldsymbol{A}$ with $\widetilde{\boldsymbol{A}}$}

In the variational principle $\mathcal{P}$ in equation (4.3), the independent variables are the displacement $\mathbf{u}$ and the overall potential A. One, however, could instead use as independent variables the displacement $\mathbf{u}$ and the perturbation vector potential $\widetilde{\mathbf{A}}$ as the later has been defined in (3.2). For this, one has to use the definitions in (A.9), such that

$$
\begin{aligned}
\mathcal{P}(\overline{\mathbf{F}}, \overline{\mathbf{B}}) & =\frac{1}{\left|\mathbf{q} \mathcal{V}_{0}^{\#}\right|} \int_{\left|\mathbf{q} \mathcal{V}_{0}^{\#}\right|}\left(\rho_{0}^{(r)} \Phi^{(r)}(\mathbf{F}, \mathbf{B})+\frac{1}{2 \mu_{0} J} \mathbf{F} \mathbf{B} \cdot \mathbf{F} \mathbf{B}\right) \mathrm{d} V-\frac{1}{2 \mu_{0} \bar{J}} \overline{\mathbf{F}} \overline{\mathbf{B}} \cdot \overline{\mathbf{F}} \overline{\mathbf{B}}+\frac{\bar{J}}{2 \mu_{0} \xi}\left|\bar{J}^{-1} \overline{\mathbf{F}} \overline{\mathbf{B}}-\mathbf{b}_{a}\right|^{2} \\
& =\frac{1}{\left|\mathbf{q} \mathcal{V}_{0}^{\#}\right|} \int_{\left|\mathbf{q} \mathcal{V}_{0}^{\#}\right|}\left(\rho_{0}^{(r)} \phi^{(r)}(\mathbf{F}, \mathbf{b})+\frac{J}{2 \mu_{0}} \mathbf{b} \cdot \mathbf{b}\right) \mathrm{d} V-\frac{\bar{J}}{2 \mu_{0}} \overline{\mathbf{b}} \cdot \overline{\mathbf{b}}+\frac{\bar{J}}{2 \mu_{0} \xi}\left|\overline{\mathbf{b}}-\mathbf{b}_{a}\right|^{2}=\mathcal{P}_{b}(\overline{\mathbf{F}}, \overline{\mathbf{b}}) . \quad \text { (A.10) }
\end{aligned}
$$

By additive decomposition of $\mathbf{b}=\overline{\mathbf{b}}+\widetilde{\mathbf{b}}$ and by taking the limit of $\overline{\mathbf{b}} \rightarrow \mathbf{b}_{a}$ in the above expression, one gets an equivalent principle that reads

$$
\mathcal{P}_{b}(\overline{\mathbf{F}}, \overline{\mathbf{b}})=\min _{\mathbf{u} \in \mathcal{K}(\overline{\mathbf{F}})} \min _{\widetilde{\mathbf{A}} \in \widetilde{\mathcal{A}}(\overline{\mathbf{b}})} \frac{1}{\left|\mathbf{q} \mathcal{V}_{0}^{\#}\right|} \int_{\left|\mathbf{q} \mathcal{V}_{0}^{\#}\right|}\left(\rho_{0}^{(r)} \phi^{(r)}\left(\mathbf{F}, J^{-1} \mathbf{F} \widetilde{\mathbf{B}} ; \overline{\mathbf{b}}\right)+\frac{1}{2 \mu_{0} J} \mathbf{F} \widetilde{\mathbf{B}} \cdot \mathbf{F} \widetilde{\mathbf{B}}\right) \mathrm{d} V
$$

where use has been made of the fact that $\int_{\left|\mathbf{q} \mathcal{V}_{0}^{\# \mid}\right|} J \overline{\mathbf{b}} \cdot \widetilde{\mathbf{b}} \mathrm{d} V=0$ as a direct result of equation (A.9). The constraint set $\mathcal{K}$ has been defined in (3.6) while

$$
\widetilde{\mathcal{A}}(\overline{\mathbf{b}})=\left\{\mathbf{b}: \exists \mathbf{x}=\chi(\mathbf{X}) \text { with } \mathbf{b}=\overline{\mathbf{b}}+\widetilde{\mathbf{b}}, \quad J \mathbf{F}^{-1} \widetilde{\mathbf{b}}=\widetilde{\mathbf{B}}=\text { Curl } \widetilde{\mathbf{A}}, \quad \widetilde{\mathbf{A}} \text { periodic, } \overline{\mathbf{b}}=\mathbf{b}_{a} \text { in } \mathbf{q} \mathcal{V}_{0}^{\#}\right\},
$$

Note also that the definition (A.11) is consistent with the average traction condition in (3.20) or (3.21) and clearly indicates that in a magnetomechanical problem the homogenization procedure should take out the term (4.1), giving rise to average Maxwell stresses due to the surrounding magnetoelastic medium.

\section{A.2. Replace independent variable $\boldsymbol{A}$ with $\widetilde{\boldsymbol{A}}$ and $\boldsymbol{m}$}

In the following, we use relation (A.5) to rewrite the augmented potential $\mathcal{P}$ in (4.3) as (the relevant "min" arguments are omitted for clarity)

$$
\begin{aligned}
\mathcal{P}(\overline{\mathbf{F}}, \overline{\mathbf{B}}) & =\frac{1}{\left|\mathbf{q} \mathcal{V}_{0}^{\#}\right|} \int_{\left|\mathbf{q} \mathcal{V}_{0}^{\#}\right|}\left(\rho_{0}^{(r)} \Phi^{(r)}(\mathbf{F}, \mathbf{B})+\frac{1}{2 \mu_{0} J} \mathbf{F} \mathbf{B} \cdot \mathbf{F} \mathbf{B}\right) \mathrm{d} V-\frac{1}{2 \mu_{0} \bar{J}} \overline{\mathbf{F}} \overline{\mathbf{B}} \cdot \overline{\mathbf{F}} \overline{\mathbf{B}}+\frac{\bar{J}}{2 \mu_{0} \xi}\left|\bar{J}^{-1} \overline{\mathbf{F}} \overline{\mathbf{B}}-\mathbf{b}_{a}\right|^{2} \\
& =\frac{1}{\left|\mathbf{q} \mathcal{V}_{0}^{\#}\right|} \int_{\left|\mathbf{q} \mathcal{V}_{0}^{\#}\right|}\left(\rho_{0}^{(r)} \psi^{(r)}(\mathbf{F}, \mathbf{m})+\frac{J \mu_{0}}{2} \mathbf{h} \cdot \mathbf{h}\right) \mathrm{d} V-\frac{1}{2 \mu_{0} \bar{J}} \overline{\mathbf{F}} \overline{\mathbf{B}} \cdot \overline{\mathbf{F}} \overline{\mathbf{B}}+\frac{\bar{J}}{2 \mu_{0} \xi}\left|\bar{J}^{-1} \overline{\mathbf{F}} \overline{\mathbf{B}}-\mathbf{b}_{a}\right|^{2}
\end{aligned}
$$

Using the results $\mathbf{h}=\mathbf{b} / \mu_{0}-\mathbf{m}, \bar{J}^{-1} \overline{\mathbf{F B}}=\overline{\mathbf{b}}$, and $\overline{\mathbf{m}}=\left(\overline{\mathbf{b}}-\mathbf{b}_{a}\right) /\left(\mu_{0} \xi\right)$ obtained in equations (2.1), (3.3) and (4.7), respectively, one gets

$$
\begin{aligned}
& \frac{1}{\left|\mathbf{q} \mathcal{V}_{0}^{\#}\right|} \int_{\left|\mathbf{q} \mathcal{V}_{0}^{\#}\right|}\left(\rho_{0}^{(r)} \psi^{(r)}(\mathbf{F}, \mathbf{m})+\frac{J}{2 \mu_{0}}\left|\overline{\mathbf{b}}+\widetilde{\mathbf{b}}-\mu_{0} \mathbf{m}\right|^{2}\right) \mathrm{d} V-\frac{1}{\left|\mathbf{q} \mathcal{V}_{0}^{\#}\right|} \int_{\left|\mathbf{q} \mathcal{V}_{0}^{\#}\right|} \frac{J}{2 \mu_{0}} \overline{\mathbf{b}} \cdot \overline{\mathbf{b}} \mathrm{d} V+\frac{\bar{J}}{2} \overline{\mathbf{m}} \cdot\left(\overline{\mathbf{b}}-\mathbf{b}_{a}\right)= \\
& \frac{1}{\left|\mathbf{q} \mathcal{V}_{0}^{\#}\right|} \int_{\left|\mathbf{q} \mathcal{V}_{0}^{\#}\right|}\left(\rho_{0}^{(r)} \psi^{(r)}(\mathbf{F}, \mathbf{m})+\frac{J}{2 \mu_{0}}\left[\widetilde{\mathbf{b}}-\left.\mu_{0} \mathbf{m}\right|^{2}-2 \mu_{0} \overline{\mathbf{b}} \cdot \mathbf{m}\right]\right) \mathrm{d} V+\frac{\bar{J}}{2} \overline{\mathbf{m}} \cdot\left(\overline{\mathbf{b}}-\mathbf{b}_{a}\right)= \\
& \frac{1}{\left|\mathbf{q} \mathcal{V}_{0}^{\#}\right|} \int_{\left|\mathbf{q} \mathcal{V}_{0}^{\#}\right|}\left(\rho_{0}^{(r)} \psi^{(r)}(\mathbf{F}, \mathbf{m})-J \mathbf{m} \cdot \overline{\mathbf{b}}+\frac{J}{2 \mu_{0}}\left|\widetilde{\mathbf{b}}-\mu_{0} \mathbf{m}\right|^{2}\right) \mathrm{d} V+\frac{\bar{J}}{2} \overline{\mathbf{m}} \cdot\left(\overline{\mathbf{b}}-\mathbf{b}_{a}\right) .
\end{aligned}
$$


where use has been made again of the fact that $\int_{\left|\mathbf{q} \mathcal{V}_{0}^{\#}\right|} J \overline{\mathbf{b}} \cdot \widetilde{\mathbf{b}} \mathrm{d} V=0{ }^{6}$ as a direct result of equation (A.9).

In this last relation, we can readily identify $\overline{\mathbf{b}}=\mathbf{b}_{a}=\mu_{0} \widehat{\mathbf{h}}_{0}$ in the notation used by Kankanala and Triantafyllidis (2004) and thus by taking the limit of $\overline{\mathbf{b}} \rightarrow \mathbf{b}_{a}$, one gets the variational principle proposed by these authors, i.e.,

$\mathcal{P}_{m}(\overline{\mathbf{F}}, \overline{\mathbf{m}}, \overline{\mathbf{b}})=\min _{\mathbf{u} \in \mathcal{K}(\overline{\mathbf{F}})} \min _{\widetilde{\mathbf{A}} \in \widetilde{\mathcal{A}}(\overline{\mathbf{b}})} \min _{\mathbf{m}} \frac{1}{\left|\mathbf{q} \mathcal{V}_{0}^{\#}\right|} \int_{\left|\mathbf{q} \mathcal{V}_{0}^{\#}\right|}\left(\rho_{0}^{(r)} \psi^{(r)}(\mathbf{F}, \mathbf{m})-J \mu_{0} \mathbf{m} \cdot \widehat{\mathbf{h}}_{0}+\frac{J}{2 \mu_{0}}\left|J^{-1} \mathbf{F} \widetilde{\mathbf{B}}-\mu_{0} \mathbf{m}\right|^{2}\right) \mathrm{d} V$

since the last term in equation (A.14) is identically zero in this limit. Note that $\mathbf{m}$ is not subjected to any particular constraint set, which can prove useful in cases that one needs to do approximations such as in the context of analytical homogenization where one can directly chose piecewise constant magnetizations in each phase similar to the corresponding case in mechanics as introduced originally by Talbot and Willis (1985). Furthermore, $\widehat{\mathbf{h}}_{0}$ is not the average $h$-field but is simply a measure related to the average $\overline{\mathbf{b}}=\mu_{0} \widehat{\mathbf{h}}_{0}$ field. In equation (A.15), the constraint sets $\mathcal{K}$ and $\widetilde{\mathcal{A}}$ have been defined in (3.6) and (A.12), respectively.

This proves the equivalence of the present formulation (4.3) which derives directly from the variational principle of Dorfmann and Ogden (2003) with that of Kankanala and Triantafyllidis (2004) and that of James and Kinderlehrer (1993) and Liu et al. (2006) if one identifies the last term in (A.15) with the perturbation $h$-field, i.e., $J^{-1} \mathbf{F} \widetilde{\mathbf{B}}-\mu_{0} \mathbf{m}=\widetilde{\mathbf{h}}=-\operatorname{grad} \phi$ (with $\phi$ denoting here the scalar potential). Again, the equivalence between (4.3) and (A.15) is true only if the functions $\phi^{(r)}(\mathbf{F}, \mathbf{b})$ and $\psi^{(r)}(\mathbf{F}, \mathbf{m})$ are obtained by the Legendre transform operations discussed in the context of equations (A.1)-(A.5).

\section{B. Application of Periodic Boundary conditions in 2D}

In this section, we discuss the application of the periodic boundary conditions in two dimensions and a plane-strain setting with the applied magnetic and mechanical fields in the plane $1-2$. The application of the proposed variational principle in 3D requires the addition of a Coulomb gauge term (Bíró and Preis, 1984; Stark et al., 2015; Sfyris et al., 2016) and will be presented elsewhere. To facilitate the discussion, we introduce in Fig.18 the two-dimensional unit cell but the following analysis can be readily generalized to three dimensional unit cells (Mbiakop et al., 2015). In this case, the subscripts in equation (3.2) go from $i, j=1,2$ and, specifically, the magnetic vector potential $\mathbf{A}$ reduces to a single scalar, i.e., the only nonzero component of $\mathbf{A}$ can be written by use of definition (3.2) as

$$
A_{1}=A_{2}=0, \quad A_{3} \equiv \alpha\left(X_{1}, X_{2}\right) \quad \rightarrow \quad \alpha\left(X_{1}, X_{2}\right)=\varepsilon_{i j} \bar{B}_{i} X_{j}+\widetilde{\alpha}\left(X_{1}, X_{2}\right),
$$

where $\widetilde{\alpha}$ is a periodic scalar function with zero mean value in the interval of the unit cell. This is a direct consequence of the fact that in the present two-dimensional problem, the components of the magnetic potential are of course independent of the $X_{3}$ direction, such that $B_{1}=B_{1}\left(X_{1}, X_{2}\right), B_{2}=B_{2}\left(X_{1}, X_{2}\right)$ and $B_{3}=0$.

In the sequel, denote the corner nodes of the square unit cell by points with coordinates $P 1=(0,0)$, $P 2=\left(L_{1}, 0\right), P 3=\left(L_{1}, L_{2}\right)$ and $P 4=\left(0, L_{2}\right)$, where $L_{1}$ and $L_{2}$ are the side lengths of the unit cell. For

\footnotetext{
${ }^{6}$ As has already been discussed by Bustamante et al. (2008), in the original work of Kankanala and Triantafyllidis (2004), the later authors have stated that the perturbation scalar potential related to the $\mathbf{h}$ field goes to zero at infinity, which in the present case would imply that $\int_{\left|\mathbf{q} \mathcal{V}_{0}^{\#}\right|} \frac{J}{\mu_{0}} \overline{\mathbf{b}} \cdot\left(\widetilde{\mathbf{b}}-\mu_{0} \mathbf{m}\right) \mathrm{d} V=0$ (since $\mu_{0} \widetilde{\mathbf{h}}=\widetilde{\mathbf{b}}-\mu_{0} \mathbf{m}$ ). This is of course not true since due to the periodic boundary conditions in equation (A.9) one has $\int_{\left|\mathbf{q} \mathcal{V}_{0}^{\#}\right|} \frac{J}{\mu_{0}} \overline{\mathbf{b}} \cdot \widetilde{\mathbf{b}} \mathrm{d} V=0$ and thus this would lead to the average magnetization being zero by $\int_{\left|\mathbf{q} \mathcal{V}_{0}^{\#}\right|} J \overline{\mathbf{b}} \cdot \mathbf{m d} V=\bar{J} \mu_{0} \overline{\mathbf{b}} \cdot \overline{\mathbf{m}}=0$, taking into account of course that $\overline{\mathbf{b}}$ and $\overline{\mathbf{m}}$ are not orthogonal vectors. In a subsequent step then they have correctly added the term $\overline{\mathbf{b}} \cdot \mathbf{m}$ as an external power term, thus obtaining the correct final variational principles in equations (3.11) and (3.12) in Kankanala and Triantafyllidis (2004). Nonetheless, this term derives simply by the fact that $\int_{\left|\mathbf{q} \mathcal{V}_{0}^{\#}\right|} J \overline{\mathbf{b}} \cdot \mathbf{m d} V \neq 0$ and by introducing the notation $\overline{\mathbf{b}}=\mu_{0} \mathbf{h}_{0}$ in the notation of Kankanala and Triantafyllidis (2004).
} 


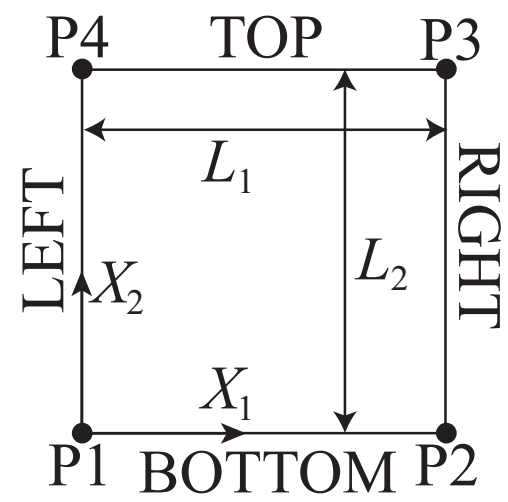

Figure 18: A sketch of a standard two dimensional square unit cell .

simplicity with the following periodicity conditions one can define the node sets 'TOP', 'BOTTOM', 'RIGHT' and 'LEFT' which contain the nodes of each of the sides of the unit cell without including the corner nodes $P 1, P 2, P 3$ and $P 4$. Next we define the number of the nodes at each of the sides as $N_{T O P}=N_{\text {ВОтTOM }}$ and $N_{R I G H T}=N_{L E F T}$.

By definition, one then can write for the periodic/perturbation fields that

$$
\begin{array}{lll}
\widetilde{\mathbf{u}}^{T O P}=\widetilde{\mathbf{u}}^{\text {BOTTOM }}, & \widetilde{\mathbf{u}}^{R I G H T}=\widetilde{\mathbf{u}}^{L E F T}, & \widetilde{\mathbf{u}}^{P 1}=\widetilde{\mathbf{u}}^{P 2}=\widetilde{\mathbf{u}}^{P 3}=\widetilde{\mathbf{u}}^{P 4}, \\
\widetilde{\alpha}^{T O P}=\widetilde{\alpha}^{\text {BOTTOM }}, & \widetilde{\alpha}^{R I G H T}=\widetilde{\alpha}^{L E F T}, & \widetilde{\alpha}^{P 1}=\widetilde{\alpha}^{P 2}=\widetilde{\alpha}^{P 3}=\widetilde{\alpha}^{P 4},
\end{array}
$$

since $\widetilde{\mathbf{u}}$ and $\widetilde{\alpha}$ are periodic fields with zero mean value.

Next, it is important that we fix the node $A$ (i.e. the origin) so that the displacement and magnetic vector field at this node is $\mathbf{u}^{P 1}=\mathbf{0}$ and $\alpha^{P 1}=\mathbf{0}$ in order to eliminate rigid body motion and zero magnetic modes, respectively, in the finite element (FE) calculations. Note that since the material is periodic (i.e., infinite), any one node in the unit cell can be fixed. Nonetheless, the choice of $P 1$ or any other corner node will allow to transform the periodic problem to a standard boundary value problem and thus simplify significantly the application of periodic boundary conditions in any commercial or non-commercial FE code.

Subsequently, one can subtract the nodal displacements of opposite boundary sides so that we get the following nodal constraints for the corner nodes by making use of (B.2) and the fact that node $P 1$ has been chosen to be fixed, i.e.,

$$
u_{i}^{P 1}=0, \quad i=1,2, \quad \text { and } \quad \alpha^{P 1}=0,
$$

and hence

$$
\begin{array}{ll}
u_{i}^{P 2}-u_{i}^{P 1}=\left(\bar{F}_{i 1}-\delta_{i 1}\right) L_{1} \Rightarrow u_{i}^{P 2}=\left(\bar{F}_{i 1}-\delta_{i 1}\right) L 1, & i=1,2 \\
u_{i}^{P 4}-u_{i}^{P 1}=\left(\bar{F}_{i 2}-\delta_{i 2}\right) L_{2} \Rightarrow u_{i}^{P 4}=\left(\bar{F}_{i 2}-\delta_{i 2}\right) L_{2}, & i=1,2 \\
\alpha^{P 2}-\alpha^{P 1}=-\bar{B}_{2} L_{1} \Rightarrow \alpha^{P 2}=-\bar{B}_{2} L_{1}, & \\
\alpha^{P 4}-\alpha^{P 1}=\bar{B}_{1} L_{2} \Rightarrow \alpha^{P 4}=\bar{B}_{1} L_{2} . &
\end{array}
$$

The above simple relations show that the displacement and magnetic components of the nodes $P 2$ and $P 4$ are one-to-one connected to certain components of the average deformation gradient $\overline{\mathbf{F}}$ and average magnetic field $\overline{\mathbf{B}}$. Then, one can write the constraint equations for the rest of the nodes making use of the above result (B.4), as

$$
\begin{aligned}
& u_{i}^{P 3}-u_{i}^{P 4}=\left(\bar{F}_{i 1}-\delta_{i 1}\right) L_{1} \Rightarrow u_{i}^{P 3}-u_{i}^{P 4}-u_{i}^{P 2}=0 \\
& u_{i}^{R I G H T}-u_{i}^{L E F T}=\left(\bar{F}_{i 1}-\delta_{i 1}\right) L_{1} \Rightarrow u_{i}^{R I G H T}-u_{i}^{L E F T}-u_{i}^{P 2}=0 \\
& u_{i}^{T O P}-u_{i}^{B O T T O M}=\left(\bar{F}_{i 2}-\delta_{i 2}\right) L_{2} \Rightarrow u_{i}^{T O P}-u_{i}^{B O T T O M}-u_{i}^{P 4}=0,
\end{aligned}
$$


and

$$
\begin{aligned}
& \alpha^{P 3}-\alpha^{P 4}=-\bar{B}_{2} L_{1} \Rightarrow \alpha^{P 3}-\alpha^{P 4}-\alpha^{P 2}=0 \\
& \alpha^{R I G H T}-\alpha^{L E F T}=-\bar{B}_{2} L_{1} \Rightarrow \alpha^{R I G H T}-\alpha^{L E F T}-\alpha^{P 2}=0, \\
& \alpha^{T O P}-\alpha^{\text {BOTTOM }}=\bar{B}_{1} L_{2} \Rightarrow \alpha^{T O P}-\alpha^{\text {BOTTOM }}-\alpha^{P 4}=0 .
\end{aligned}
$$

The above algebraic analysis reveals that all periodic constraints between all nodes can be written in terms of the degrees of freedom of two only nodes (three in the case of $3 \mathrm{D})$, i.e., $\left(\mathbf{u}^{P 2}, \mathbf{u}^{P 4}\right)$ and $\left(\alpha^{P 2}, \alpha^{P 4}\right)$, which, in turn, are given in terms of $\overline{\mathbf{F}}$ and $\overline{\mathbf{B}}$, respectively by equation (B.4). This, further, implies that the only nodes that boundary conditions need to be applied are $P 2$ and $P 4$ (together with the $P 1$ which is fixed). Therefore, the application of the average deformation gradient $\overline{\mathbf{F}}$ and average magnetic field $\overline{\mathbf{B}}$ is done by simply applying standard boundary conditions (see (B.4)) at the relevant nodes together with the rest of the (B.5). These constraints are linear and can be applied via elimination, Lagrange multipliers or penalty methods.

In the context of the augmented variational principle (4.3), we do not apply directly the Lagrangian magnetic field $\overline{\mathbf{B}}$ but the Eulerian one $\overline{\mathbf{b}}$. The condition (4.2) is a nonlinear constraint for $\overline{\mathbf{B}}$ in terms of the given $\overline{\mathbf{b}}$ and the average deformation gradient $\overline{\mathbf{F}}$, which is applied via the last penalty energy term in (4.3). Since $\overline{\mathbf{B}}$ is a direct function of the nodal degrees of freedom, $\left(\alpha^{P 2}, \alpha^{P 4}\right)$, as shown in equation (B.4), it suffices to add the contribution of the last penalty term in (4.3) to the overall force vector and stiffness matrix by considering the first and second variations with respect to the relevant degrees of freedom of the nodes $P 2$ and $P 4$. This is a straightforward operation and is not discussed further.

\section{References}

Belytschko, T., Lu, Y.Y., Gu, L., 1994. Element-free galerkin methods. International Journal for Numerical Methods in Engineering 37, 229-256.

Bertoldi, K., Boyce, M., Deschanel, S., Prange, S., Mullin, T., 2008. Mechanics of deformation-triggered pattern transformations and superelastic behavior in periodic elastomeric structures. Journal of the Mechanics and Physics of Solids 56, $2642-2668$.

Bertoldi, K., Reis, P.M., Willshaw, S., Mullin, T., 2010. Negative poisson's ratio behavior induced by an elastic instability. Advanced Materials 22, 361-366.

Bíró, O., Preis, K., 1984. On the use of the magnetic vector potential in the finite element analysis of three-dimensional eddy currents. IEEE TRANSACTIONS ON MAGNETICS 25, 3145.

Brown, W.F., 1966. Magnetoelastic interactions. Springer-Verlag, New York.

Bustamante, R., 2010. Transversely isotropic nonlinear magneto-active elastomers. Acta Mechanica 210, 183-214.

Bustamante, R., Dorfmann, A., Ogden, R.W., 2008. On variational formulations in nonlinear magnetoelastostatics. Mathematics and Mechanics of Solids 13. http://mms.sagepub.com/content/early/2007/09/10/1081286507079832.full.pdf+html.

Bustamante, R., Dorfmann, A., Ogden, R.W., 2009. Nonlinear electroelastostatics: a variational framework. Zeitschrift für angewandte Mathematik und Physik 60, 154-177.

Chi, H., Talischi, C., Lopez-Pamies, O., Paulino, G.H., 2016. A paradigm for higher-order polygonal elements in finite elasticity using a gradient correction scheme. Computer Methods in Applied Mechanics and Engineering, --

Cohen, N., Dayal, K., deBotton, G., 2016. Electroelasticity of polymer networks. Journal of the Mechanics and Physics of Solids 92, $105-126$.

Danas, K., Kankanala, S., Triantafyllidis, N., 2012. Experiments and modeling of iron-particle-filled magnetorheological elastomers. Journal of the Mechanics and Physics of Solids 60, $120-138$.

Danas, K., Triantafyllidis, N., 2014. Instability of a magnetoelastic layer resting on a non-magnetic substrate. Journal of the Mechanics and Physics of Solids 69, $67-83$.

De Simone, A., 1993. Energy minimizers for large ferromagnetic bodies. Archive for Rational Mechanics and Analysis 125, 99-143.

Diguet, G., Beaugnon, E., Cavaillé, J., 2010. Shape effect in the magnetostriction of ferromagnetic composite. J. Magn. Magn. Mater. 322, $3337-3341$.

Dorfmann, A., Ogden, R., 2005. Some problems in nonlinear magnetoelasticity. Zeitschrift für angewandte Mathematik und Physik ZAMP 56, 718-745.

Dorfmann, A., Ogden, R.W., 2003. Magnetoelastic modelling of elastomers. European J. Mech. A/Solids 22, $497-507$.

Ericksen, J.L., 2006. A modified theory of magnetic effects in elastic materials. Mathematics and Mechanics of Solids 11, 23-47. http://mms. sagepub.com/content/11/1/23.full.pdf+html.

Eshelby, J., 1957. The determination of the elastic field of an ellipsoidal inclusion and related problems. Proc. R. Soc. Lond. A $241,376-396$.

Galipeau, E., Ponte Castañeda, P., 2013. A finite-strain constitutive model for magnetorheological elastomers: Magnetic torques and fiber rotations. Journal of the Mechanics and Physics of Solids 61, 1065 - 1090. 
Galipeau, E., Rudykh, S., deBotton, G., Ponte Castañeda, P., 2014. Magnetoactive elastomers with periodic and random microstructures. International Journal of Solids and Structures 51, $3012-3024$.

Gerbal, F., Wang, Y., Lyonnet, F., Bacri, J.C., Hocquet, T., Devaud, M., 2015. A refined theory of magnetoelastic buckling matches experiments with ferromagnetic and superparamagnetic rods. Proceedings of the National Academy of Sciences 112, 7135-7140. http://www.pnas.org/content/112/23/7135.full.pdf.

Ginder, J., Nichols, M., Elie, L., Tardiff, J., 1999. Magnetorheological elastomers: properties and applications. Smart Structures and Materials 1999: Smart Materials Technologies Ed. by M. Wuttig, Proc. of SPIE. 3675, $131-138$.

Henann, D.L., Chester, S.A., Bertoldi, K., 2013. Modeling of dielectric elastomers: Design of actuators and energy harvesting devices. Journal of the Mechanics and Physics of Solids 61, $2047-2066$.

Huang, S., Pessot, G., Cremer, P., Weeber, R., Holm, C., Nowak, J., Odenbach, S., Menzel, A.M., Auernhammer, G.K., 2016. Buckling of paramagnetic chains in soft gels. (Paper) Soft Matter 12, 228-237.

James, R., Kinderlehrer, D., 1993. Theory of magnetostriction with applications to tbxdy1xfe2. Phil. Mag. B 68, 237-274.

Javili, A., Chatzigeorgiou, G., Steinmann, P., 2013. Computational homogenization in magneto-mechanics. International Journal of Solids and Structures 50, $4197-4216$.

Kalina, K.A., Metsch, P., Kästner, M., 2016. Microscale modeling and simulation of magnetorheological elastomers at finite strains: A study on the influence of mechanical preloads. International Journal of Solids and Structures 102-103, 286 - 296.

Kankanala, S.V., Triantafyllidis, N., 2004. On finitely strained magnetorheological elastomers. J. Mech. Phys. Solids 52, 2869 $-2908$.

Keip, M.A., Rambausek, M., 2015. A multiscale approach to the computational characterization of magnetorheological elastomers. International Journal for Numerical Methods in Engineering Nme.5178.

Landis, C.M., 2002. A new finite-element formulation for electromechanical boundary value problems. International Journal for Numerical Methods in Engineering 55, 613-628.

Lefèvre, V., Lopez-Pamies, O., 2015. The overall elastic dielectric properties of fiber-strengthened/weakened elastomers. Journal of Applied Mechanics 82 (11).

Lefèvre, V., Lopez-Pamies, O., 2016a. Nonlinear electroelastic deformations of dielectric elastomer composites: \{I\} — ideal elastic dielectrics. Journal of the Mechanics and Physics of Solids, -.

Lefèvre, V., Lopez-Pamies, O., 2016b. Nonlinear electroelastic deformations of dielectric elastomer composites: \{II\} - nongaussian elastic dielectrics. Journal of the Mechanics and Physics of Solids , - .

Liu, L., 2014. An energy formulation of continuum magneto-electro-elasticity with applications. Journal of the Mechanics and Physics of Solids 63, $451-480$.

Liu, L., James, R., Leo, P., 2006. Magnetostrictive composites in the dilute limit. Journal of the Mechanics and Physics of Solids 54, $951-974$.

López Jiménez, F., 2014. Modeling of soft composites under three-dimensional loading. Composites: Part B 59, $173-180$.

Lopez-Pamies, O., 2014. Elastic dielectric composites: Theory and application to particle-filled ideal dielectrics. Journal of the Mechanics and Physics of Solids 64, $61-82$.

Lopez-Pamies, O., Goudarzi, T., Danas, K., 2013. The nonlinear elastic response of suspensions of rigid inclusions in rubber: Ii - a simple explicit approximation for finite-concentration suspensions. Journal of the Mechanics and Physics of Solids 61 , $19-37$.

Martin, J.E., Anderson, R.A., Read, D., Gulley, G., 2006. Magnetostriction of field-structured magnetoelastomers. Phys. Rev. E 74, 051507 .

Mbiakop, A., Constantinescu, A., Danas, K., 2015. An analytical model for porous single crystals with ellipsoidal voids. Journal of the Mechanics and Physics of Solids 84, 436-467.

Metsch, P., Kalina, K.A., Spieler, C., Kästner, M., 2016. A numerical study on magnetostrictive phenomena in magnetorheological elastomers. Computational Materials Science 124, $364-374$.

Michel, J., Lopez-Pamies, O., Ponte Castañeda, P., Triantafyllidis, N., 2007. Microscopic and macroscopic instabilities in finitely strained porous elastomers. Journal of the Mechanics and Physics of Solids 55, $900-938$.

Michel, J.C., Moulinec, H., Suquet, P., 1999. Effective properties of composite material with periodic microstructure: a computational approach. Comput. Methods Appl. Mech. Engrg. 172, 109-143.

Miehe, C., Vallicotti, D., Teichtmeister, S., 2016. Homogenization and multiscale stability analysis in finite magneto-electroelasticity. application to soft matter ee, $\{\mathrm{ME}\}$ and $\{\mathrm{MEE}\}$ composites. Computer Methods in Applied Mechanics and Engineering 300, $294-346$.

Nayroles, B., Touzot, G., Villon, P., 1992. Generalizing the finite element method: Diffuse approximation and diffuse elements. Computational Mechanics 10, 307-318.

Ponte Castañeda, P., Galipeau, E., 2011. Homogenization-based constitutive models for magnetorheological elastomers at finite strain. J. Mech. Phys. Solids 59, $194-215$.

Prall, D., Lakes, R., 1997. Properties of a chiral honeycomb with a poisson's ratio of — 1. International Journal of Mechanical Sciences 39, $305-314$

Rudykh, S., Bertoldi, K., 2013. Stability of anisotropic magnetorheological elastomers in finite deformations: A micromechanical approach. Journal of the Mechanics and Physics of Solids 61, 949 - 967.

Segurado, J., Llorca, J., 2002. A numerical approximation to the elastic properties of sphere-reinforced composites. Journal of the Mechanics and Physics of Solids 50, 2107-2121.

Sfyris, G.I., Danas, K., Wen, G., Triantafyllidis, N., 2016. Freedericksz instability for the twisted nematic device: A threedimensional analysis. Phys. Rev. E 94, 012704.

Shan, S., Kang, S.H., Wang, P., Qu, C., Shian, S., Chen, E.R., Bertoldi, K., 2014. Harnessing multiple folding mechanisms in soft periodic structures for tunable control of elastic waves. Advanced Functional Materials 24, 4935-4942. 
Spadoni, A., Ruzzene, M., Gonella, S., Scarpa, F., 2009. Phononic properties of hexagonal chiral lattices. Wave Motion 46, $435-450$.

Spieler, C., Kästner, M., Goldmann, J., Brummund, J., Ulbricht, V., 2013. Xfem modeling and homogenization of magnetoactive composites. Acta Mechanica 224, 2453-2469.

Spieler, C., Metsch, P., Kästner, M., Ulbricht, V., 2014. Microscale modeling of magnetoactive composites undergoing large deformations. TECHNISCHE MECHANIK 34, 39-50.

Stark, S., Semenov, A.S., Balke, H., 2015. On the boundary conditions for the vector potential formulation in electrostatics. International Journal for Numerical Methods in Engineering 102, 1704-1732.

Talbot, D., Willis, J., 1985. Variational principles for inhomogeneous nonlinear media. IMAJ.Appl.Math. 35, 39-54.

Talischi, C., Paulino, G.H., Pereira, A., Menezes, I.F.M., 2010. Polygonal finite elements for topology optimization: A unifying paradigm. International Journal for Numerical Methods in Engineering 82, 671-698.

Tian, L., Tevet-Deree, L., deBotton, G., Bhattacharya, K., 2012. Dielectric elastomer composites. Journal of the Mechanics and Physics of Solids 60, $181-198$

Tipton, C.R., Han, E., Mullin, T., 2012. Magneto-elastic buckling of a soft cellular solid. Soft Matter 8, 6880-6883.

Torquato, S., 2002. Random heterogeneous materials: Micorstructures and macroscopic properties. Springer .

Wang, S., Decker, M., Henann, D.L., Chester, S.A., 2016. Modeling of dielectric viscoelastomers with application to electromechanical instabilities. Journal of the Mechanics and Physics of Solids 95, $213-229$. 